\title{
Investigation of Causal Relationship between Stock Prices and Trading Volume using Toda and Yamamoto Procedure
}

\author{
Sushil BAJAJ", Vibha DUA ${ }^{* *}$
}

\begin{abstract}
The present study probes the relationship between the stock prices and trading volume. For achieving this purpose, daily data of adjusted closing stock prices, trading volume of 39 individual securities and S\&P CNX Nifty from January 1, 1998 to May 31, 2013 have been used. In this study, instead of applying ordinary Granger causality test to investigate the relationship between stock prices and trading volume, Toda and Yamamoto (1995) procedure has been applied for analyzing the data. Lag length chosen by AIC and FPE criterion has been insured by running Lagrange Multiplier (LM) test and causality determined by Toda and Yamamoto test has also been confirmed by using VAR methodology. Although, Toda and Yamamoto and VAR test produced little dissimilar results, nevertheless, the empirical analysis provides sufficient grounds to declare the presence of interaction between stock prices and trading volume.
\end{abstract}

Keywords: Stock Returns, Trading Volume, Causal Relationship, Toda and Yamamoto Procedure, Vector Autoregression

JEL Code Classification: C32, G10, G19

\footnotetext{
* Assistant Professor, Department of Commerce, CRM Jat College, Hisar (Haryana), India. Email: bajajsushil86@gmail.com

${ }^{* *}$ Assistant Professor, Institute of Marketing and Management, Qutub Institutional Area, New Delhi, India. Email: dua vibha@rediffmail.com

Copyright (C), 2014 International Ataturk Alatoo University.
} 


\section{Introduction}

The Indian stock markets exhibit greater volatility compared with many other emerging and developed markets of the world such as China, Russia, Brazil, USA etc. Volatility oscillates the volume of trade in the market which impacts stock price and ultimately the stock returns. Therefore, it becomes imperative to understand the inter-relationship between the trading volume and stock returns which may help investors to identify the future patterns of the stock market for effective decision making. Stock's price-volume relation can also be used for the formation of trading strategies for maintaining the efficiency of the stock markets. Theoretically, low volume makes the market illiquid which causes low price fluctuation whereas high volume usually implies that the market is highly liquid, resulting in high price variability that lessens the price effect of large trades. In general, with an increase in volume, broker revenue increases, and market makers have greater opportunity for profit as a result of higher turnover. Many researches have been performed worldwide on the different stock markets, especially in the USA, to investigate the relationship between stock return/price and trading volume. Karpoff (1987) summarised the importance of understanding this relationship to predict various volume-price/return relations that depend on the level of information and the extent to which market prices/volumes convey this information. Researchers in this field have scrutinized the volume-price/return relationship in a variety of contexts and by employing a range of econometric techniques. Early studies examined the correlation between volume and the price change as well as volume and the absolute value of the price change (Granger and Morgenstern (1963), Godfrey et al., (1964), Crouch (1970)). More recent studies were interested in investigating the causal relationship between these two market variables (Smirlock and Starks, 1988; Chordia and Swaminathan, 2000; Chen et al., 2001). The linear and non-linear causality between the stock prices and trading volume has also received a substantial amount of attention in the literature (Campbell et al., 1993; Martikainen et al., 1994; Hiemstra and Jones, 1994) but most of these researches have focused almost exclusively on the well-developed financial markets, usually the U.S. markets. Taking clue from the earlier studies, this study makes an attempt to empirically investigate the interaction between stock prices and trading volume by using a unique and more refined technique called Toda and Yamamoto procedure. This new and more precise econometric model on more recent data would be helpful in reaching on the conclusion regarding relationship between stock prices and trading volume in the Indian Stock Market.

\section{Literature Review}

Granger and Morgenstern (1963) were the founding members of conducting empirical analysis of volume-price relationship. They revealed that there is no correlation between prices or absolute price changes and volumes using weekly or daily transaction data for the stock market price index and for individual stocks. Karpoff (1987) proposed a simple model of the price-volume relationship called 
"asymmetric volume-price change hypothesis", showing that the relationship is fundamentally different for positive and negative price changes. Smirlock and Starks (1988) empirically examined the lagged relationship between the absolute price changes and volume in the equity markets and investigate the implications of this relationship for the microstructure of these markets. Using Granger causality tests their results indicate that there is a significant causal relationship between absolute price changes and volume at the firm level. Furthermore, relationship is stronger in periods surrounding earning announcements. Blume et al. (1994) also studied and developed a model that links trading volume to stock price behavior. The major implication of their model is that investors who focus on past trading volume can obtain additional profits and perform better return than those who use only price measures. Hiemstra and Jones (1994) used nonlinear Granger causality test to examine the nonlinear causal relation between the percentage changes in the NYSE trading volume and stock returns and found positive nonlinear bidirectional relationship between returns and volume. Bhagat and Bhatia (1996) also employed daily and weekly data to test the causal relationship between volume and return, finding return causes volume but not vice versa. Saatcioglu and Starks (1998) used monthly data taken from the six Latin American stock markets to test the relation between price changes and volume. They found a positive pricevolume and a causal relationship from volume to stock price changes but not vice versa. Chordia and Swaminathan (2000) found that past trading volume can be used to predict the future stock price momentum. Ratner and Leal (2001) examined the Latin American and Asian financial markets and found a positive contemporaneous relation between return and volume in these countries except India. At the same time they observed that there exists a bi-directional causal relation between return and volume. In summary, the return and volume are strongly related contemporaneously but there is a little evidence that either can be used to predict the other. Similarly, Llorente et al. (2002) used the simple model to explore the dynamic relation between volume and returns. According to their model, returns generated by hedging-motivated trades reverse themselves, while returns generated by speculation-motivated trades tend to continue themselves. Their empirical results support the predictions of the model on the nature of the dynamic volume-return relation. De Medeiros and Doornik (2006), Zolontoy and Melenberg (2007) and Sabri (2008) also investigated the empirical relationship between stock returns, return volatility and trading volume in the various stock markets and found the support for a contemporaneous as well as dynamic relationship between the stock returns and trading volume. Mahajan and Singh (2009) studied the relationship between return, volume and volatility dynamics by using Indian sensitivity index Sensex and evidenced the presence of sequential arrival of information due to the direction of causality from volatility to volume. Besides this, inclusion of trading volume in the $\operatorname{GARCH}(1,1)$ model slightly brought down the volatility persistence and confirmed its prominent role in the precision and dispersion of information signals. A more recent study by Mehrabanpoor et al., 
(2011) investigated the empirical relationship between the stock indices and volume in the Tehran Stock Exchange. Using monthly indices, value and turnover for the period from 2003 to 2009, they proved that there is positive relationship between exchange turnover value and stock exchange indices in the Tehran Stock Exchange. A Similar study by Tripathy (2011) explored the relationship between trading volume and stock returns using data from the Indian Stock Market during the period from January 2005 to January 2010. By using Bivariate Regression model, VECM Model, VAR, IRF and Johansen's cointegration test, his findings support the existence of significant contemporaneous relationship between return volatility and trading volume indicating that information may flow simultaneously rather than sequentially into the market. Moreover, the study also found that the trading volume is associated with an increase in return volatility and that this relationship is asymmetrical. This implied that daily new information in the market may have a significant impact on price volatility, which indicates that the bad news generates more impact on volatility of the stock return and trading volume. Darwish (2012) studied the causal relationship between return and trading volume in the Palestine Stock Exchange. The author has used weekly trading volume and returns over the period from October 2000 to August 2010 and employed GARCH $(1,1)$ model to test the existence of the positive contemporaneous relationship. The study found that the relationship preserves after taking heteroskedasticity into account. Moreover, the results of the Granger causality test show that there is bidirectional Granger causality between returns and trading volume regardless of the measures of trading volume used.

\section{Research Methodology}

\subsection{Data Source and Time Period}

The National Stock Exchange was incorporated in the year 1992 and is a well diversified 50 stock index answering for 22 sectors of the economy. It is used for a variety of purposes such as benchmarking fund portfolios, index based derivatives and index funds and promoted by the leading financial institutions. Due to the NSE's tremendous growth (in terms of turnover as well as number of contracts) and its 'CCR AAR' rating assigned by CRISIL ${ }^{1}$, present study uses the historical data of NSE. As far as financial market is concerned, investors immediately react (buying/selling) on arrival of new information and trading volume is the result of buying and selling which eventually impacts the security prices. The main objective of the present study is to identify the interaction between stock prices and trading volume and to accomplish the same, adjusted closing prices and trading volume data of 39 individual securities and S\&P CNX Nifty for the period from January 1, 1998 to May 31, 2013 have been taken into account. It is pertinent to mention here that only those securities have been considered wherein trading had started on or

\footnotetext{
${ }^{1}$ CNX Nifty. Retrieved from http://www.nseindia.com/products/content/equities/indices/cnx_nifty.htm as on December 28, 2013.
} 
before the year 2005 and the complete dataset have been retrieved from the renowned database CMIE PROWESS. Data collected from the above mentioned sources have been analyzed using the Toda and Yamamoto Procedure. Steps for applying the Toda and Yamamoto procedure are as follows:

The returns of the stock prices are calculated as given in equation (1) below:

$R_{t}=\ln \left(P_{t} / P_{t-1}\right)$

In Equation (1), $\ln P_{t}$ stands for natural logarithm of the present closing price of securities and $\ln P_{t-1}$ is the natural logarithm of the previous day's closing prices. Trading volume is used to depict the amount of buying and selling that has taken place at particular price. For the present study, instead of considering raw values of trading volumes, their logarithm has been considered to enhance normality in the data by following the equation (2).

$V_{t}=\ln \operatorname{Vol}_{t}$

In Equation (2), $\ln \mathrm{Vol}_{t}$ stands for natural logarithm of the shares traded (i.e. volume) at time t.

\subsection{Unit Root Test}

The present study deals with the time series data that is generally found nonstationary and presence of the same makes hypothesis tests' results undependable. Existence of non stationarity or unit root in the dataset is checked by practicing ADF (Augmented Dickey Fuller) and PP (Phillips-Perron) tests. Appropriate lag length for the above mentioned two tests is determined by following the $\mathrm{AIC}$ and $\mathrm{SIC}$ criterions.

\subsection{Toda and Yamamoto Procedure}

This section of the study highlights the econometric technique used to study the causal relationship between the volume and closing prices. Ordinarily, a statistical approach proposed by Clive W Granger (1969) known as Granger causality is used to infer cause and effect relationship between the two (or more) time series. The test uses the following Vector Autoregression (VAR) model: -

$P_{i}=\alpha_{0}+\sum_{i=1}^{n} \alpha_{i} V_{t-i}+\sum_{j=1}^{n} \beta_{j} P_{t-j}+\mu_{1 t}$

$V_{i}=\delta_{0}+\sum_{i=1}^{n} \delta_{i} V_{t-i}+\sum_{j=1}^{n} \gamma_{j} P_{t-j}+\mu_{2 t}$

In Equation (3), $P_{i}$ is the log normal daily closing price series and $V_{i}$ is the log normal daily traded volume. $\alpha_{i}, \beta_{j}, \delta_{i}, \gamma_{j}$ are the coefficients of the respective variables and $\mu_{1 t}, \mu_{2 t}$ are the error terms assumed to be uncorrelated. Causality from volume to closing prices and closing prices to volume is confirmed if the estimated coefficient on the lagged $V_{i}$ and $P_{i}$ are found statistically different from zero(i.e. $\sum \alpha_{i}=\sum \gamma_{j} \neq 0$ ). The Granger causality test is conventionally run by estimating the Vector Autoregressive (VAR) models but according to Granger 
Representation Theorem, first of all cointegration test must be run if the series are I(1) or integrated of the same order as at least unidirectional causality must exist in either way but the same test fails to play eminent role if the series are not I(1) or integrated of different orders such as one is $I(N)$ and the other is different from I(N) say $\mathrm{I}(\mathrm{N}+1)$ or $\mathrm{I}(\mathrm{N}-1)$. However, Granger causality test can be conducted in an unrestricted VAR model to test the linear restrictions on the parameters of a model, usually by the help of Wald statistic (or F-statistic) but not advised by Phillips and Toda (1993) as Wald test statistics' distribution do not follow its usual asymptotic chi-square distribution and involves 'nuisance parameters' that you can't observe, and so it is totally non-standard. Besides this, Granger causality test depends critically on the number of lagged terms introduced in the model. Due to the presence of above mentioned limitations, the present study adopts a substitute model to the estimation of an unrestricted VAR, popularly known as Toda and Yamamoto (1995) procedure that guarantees the asymptotic distribution of the Wald statistic (an asymptotic $\chi^{2}$-distribution), since the testing procedure is robust to the integration and co-integration properties of the process.

\subsection{Steps involved in Toda and Yamamoto Procedure}

Toda and Yamamoto (1995) procedure includes two steps wherein first step involves the identification of the maximum order of integration for the variables in the model. For this purpose, this study uses the Augmented Dickey fuller (ADF) and Phillips-Perron (PP) tests to identify the maximum order of integration assigned a $\operatorname{symbol}\left(\rho_{\max }\right) . \rho_{\max }$ is equal to $\mathrm{N}$ if both variables in the time series are found $I(N)$ or one of the variables is found $I(N)$ and the other one is found $I(N-1)$. Present study runs ADF and PP tests by taking the lag length suggested by AIC (Akaike Information Criterion). Second step involves the determination of the appropriate maximum lag length for the variables in the $\operatorname{VAR}, \operatorname{say}(k)$ using the two usual information criterions i.e. AIC (Akaike Information Criterion) and Final Prediction Error (FPE). The motive behind choosing the appropriate lag length is to remove serial correlation. To ensure the absence of serial correlation, present study also applies Lagrange Multiplier (LM) test on the residuals for serial independence by testing (at 10 per cent level of significance) the null hypothesis of "no serial correlation" to scrutinize the authenticity of the optimal lag length chosen by using AIC and FPE criterions and increased/decreased the same; if suggested by the LM test until serial correlation issues are resolved. After the determination of preferred VAR model including optimal $\operatorname{lag}(k)$, number of lags chosen at the first step for each of the variable i.e. $\left(\rho_{\max }\right)$ is further added into each of the VAR equation that results in below mentioned bivariate VAR $\left(k+\rho_{\text {max }}\right)$ model.

$P_{t}=\phi+\sum_{i=1}^{k} \alpha_{i} P_{t-i}+\sum_{i=k+1}^{k+\rho_{\max }} \alpha_{i} P_{t-i}+\sum_{i=1}^{k} \beta_{i} V_{t-i}+\sum_{i=k+1}^{k+\rho_{\max }} \beta_{i} V_{t-i}+\mu_{1 t}$

$V_{t}=\varphi+\sum_{i=1}^{k} \delta_{i} V_{t-i}+\sum_{i=k+1}^{k+\rho_{\max }} \delta_{i} V_{t-i}+\sum_{i=1}^{k} \gamma_{i} P_{t-i}+\sum_{i=k+1}^{k+\rho_{\max }} \gamma_{i} P_{t-i}+\mu_{2 t}$ 
Where, $P_{t}$ is $\log$ normal closing price, $V_{t}$ is log normal volume, $\alpha_{i}, \beta_{i}, \delta_{i}$ and $\gamma_{i}$ are the model's parameters; $\rho_{\max }$ is the maximum order of integration expected to occur; $\mu_{1 t} \sim N\left(0, \sum_{\mu 1}\right)$ and $\mu_{2 t} \sim N\left(0, \sum_{\mu 2}\right)$ are residuals of the model. By following the above mentioned VAR model, null hypothesis $\left(H_{0}\right)$ of non causality from volume to closing price and closing price to volume expressed as: $-H_{0}: \beta_{i}=$ $0, \forall i=1,2,3 \ldots . k), \gamma_{i}=0, \forall i=1,2,3 \ldots . k$ are tested using a Modified Wald test (MWALD).

\subsection{Vector Autoregression}

Previous studies have shown the application of co-integration and VECM model for the determination of relationship between underlying variables' price and trading volume series. Though, it is a sound methodology but can be applied only if both underlying variables are non-stationary and integrated of the same order, absence of which demands the application of VAR (Vector Auto-regression) model. In the present study, closing price series are $I(1)$ whereas trading volume series are found $I(0)$ and due to the difference in their integration, VAR has been applied. For the determination of the cause and effect relationship, bivariate VAR i.e. (inclusion of only two variables) has been applied.

$$
\begin{aligned}
& R_{t}=\alpha+\sum_{j=1}^{k} \beta_{j} R_{t-j}+\sum_{j=1}^{k} \gamma_{j} V_{t-j}+\varepsilon_{1 t} \\
& V_{t}=\delta+\sum_{j=1}^{k} \theta_{j} R_{t-j}+\sum_{j=1}^{k} \varphi_{j} V_{t-j}+\varepsilon_{2 t}
\end{aligned}
$$

In Equation (7) $R_{t}$ is the security' return series and $V_{t}$ is the log normal daily traded volume. $\alpha$ and $\delta$ are intercept terms; $\beta_{j s}, \gamma_{j s}, \theta_{j s}, \varphi_{j s}$ are the coefficients of respective variables; $\varepsilon_{1 t}, \varepsilon_{2 t}$ are white noise stochastic error terms and $k$ explains the maximum lag length used for running the model. In the above model, causality from volume $\left(V_{t}\right)$ to return series $\left(R_{t}\right)$ can be witnessed only if some of the coefficients $\left(\gamma_{j}\right)$ are found significant. Similarly, causality from return series $\left(R_{t}\right)$ to volume $\left(V_{t}\right)$ is confirmed in the presence of significant coefficients $\left(\theta_{j s}\right)$. Lag length for the execution of the model is firstly chosen by following the AIC (Akaike Information Criterion) and Final Prediction Error (FPE) criterions and is further checked by running LM (Lagrange Multiplier) test. It is worthwhile to mention here that $t$-test is used to scrutinise the significance of independent variables' coefficients.

\section{Results and Interpretation}

\subsection{Descriptive Statistics}

Before moving on with the in-depth analysis, it is important to recognise the general characteristics of the data as shown in the Table 1 . Results clearly depict that except Indusind Bank, mean return of all the individual securities and S\&P CNX Nifty have positive return surrounded with reasonable amount of variation. Around 64 per cent of the individual securities are skewed to the right side whereas rest of the securities (i.e. 36 per cent) and an index are negatively skewed (i.e. skewed to 
the left) which adjudge the return series asymmetric and abnormal. Absence of normality has further been inspected by the help of $p$-values of Jarque-Bera test. The shape of the frequency distribution of the return series is leptokurtic in the present dataset because of the presence of kurtosis values above 3 that indicates the presence of positive correlation between return and volume series. On the other hand, trading volume has relatively higher volatility around its mean values for the complete dataset. Furthermore, 82 per cent of the individual securities and index volume series are negatively skewed and 39 per cent of the individual securities and trading volume have shown platykurtic shape (i.e. kurtosis < 3). As far as JB-test for volume series is concerned, it also confirms the presence of nonnormality in the complete dataset due to the rejection of the null hypothesis of normal distribution.

Table 1: Descriptive Statistics

\begin{tabular}{|c|c|c|c|c|c|c|c|}
\hline Company & & Mean & Std. Dev. & Skewness & Kurtosis & $\begin{array}{l}\text { Jarque- } \\
\text { Bera }\end{array}$ & $\begin{array}{l}\text { Probability } \\
\text { (J-B Test) }\end{array}$ \\
\hline \multirow{2}{*}{ ACC } & Return & 0.000601 & .02621 & -0.128115 & .40321 & 1872.34 & 0.00000 \\
\hline & Volume & 13.43339 & 1.04344 & 0.167461 & 2.96605 & 18.22188 & 0.00000 \\
\hline \multirow{2}{*}{ Asian Paints } & Return & 0.000956 & 0.01836 & 0.259259 & 7.96638 & 4007.082 & 0.00000 \\
\hline & Volume & 9.617747 & 1.37343 & -0.09255 & 2.78024 & 13.27132 & 0.00013 \\
\hline \multirow{2}{*}{ Axis Bank } & Return & 0.001231 & 0.03135 & 0.457583 & 7.89613 & 3728.679 & 0.00000 \\
\hline & Volume & 12.75449 & 1.70943 & -0.36324 & 2.13822 & 190.991 & 0.00000 \\
\hline \multirow{2}{*}{$\begin{array}{l}\text { Bank of } \\
\text { Baroda }\end{array}$} & Return & 0.000458 & 0.02978 & 0.060242 & 7.31856 & 2999.549 & 0.00000 \\
\hline & Volume & 12.77508 & 1.28529 & -0.61336 & 3.29530 & 255.926 & 0.00000 \\
\hline \multirow{2}{*}{ Bharti Airtel } & Return & 0.000965 & 0.02656 & 0.905525 & 12.95 & 11860.5 & 0.00000 \\
\hline & Volume & 14.50083 & 1.18082 & -0.67166 & 3.23318 & 215.6352 & 0.00000 \\
\hline \multirow{2}{*}{$\begin{array}{l}\text { Bharat } \\
\text { Petroleum }\end{array}$} & Return & 0.000346 & 0.05885 & 2.251575 & 730.482 & 84018613 & 0.00000 \\
\hline & Volume & 12.66124 & 1.51514 & -1.45569 & 6.33007 & 3106.03 & 0.00000 \\
\hline \multirow{2}{*}{ BHEL } & Return & 0.000461 & 0.03596 & 2.694753 & 291.914 & 13255734 & 0.00000 \\
\hline & Volume & 13.37121 & 0.99249 & 0.22492 & 3.42403 & 60.68456 & 0.00000 \\
\hline \multirow{2}{*}{ Cipla } & Return & 0.0008 & 0.02308 & -0.02100 & 6.74938 & 2259.501 & 0.00000 \\
\hline & Volume & 13.54549 & 1.14338 & -1.28784 & 5.42489 & 2011.67 & 0.00000 \\
\hline \multirow{2}{*}{ Dr. Reddy } & Return & 0.000843 & 0.02468 & -0.02801 & 7.78484 & 3679.882 & 0.00000 \\
\hline & Volume & 12.4012 & 0.94633 & -0.59666 & 3.80752 & 333.7411 & 0.00000 \\
\hline \multirow{2}{*}{ GAIL } & Return & 0.000334 & 0.02601 & 0.01949 & 11.7720 & 12360.22 & 0.00000 \\
\hline & Volume & 12.97749 & 2.03004 & -0.82772 & 2.83793 & 444.5271 & 0.00000 \\
\hline \multirow{2}{*}{ Grasim } & Return & 0.000547 & 0.02594 & -0.09160 & 7.7062 & 3564.814 & 0.00000 \\
\hline & Volume & 11.5859 & 1.04582 & -0.77778 & 4.87942 & 956.5398 & 0.00000 \\
\hline \multirow{2}{*}{$\begin{array}{l}\text { Gujarat } \\
\text { Ambuja }\end{array}$} & Return & 0.000596 & 0.02492 & 0.082435 & 5.41873 & 939.1721 & 0.00000 \\
\hline & Volume & 14.38616 & 1.06696 & -0.43064 & 3.58308 & 172.9101 & 0.00000 \\
\hline \multirow{2}{*}{ HCL Tech } & Return & 0.00010 & 0.03423 & -0.35525 & 7.66374 & 3090.715 & 0.00000 \\
\hline & Volume & 13.91834 & 0.89234 & 0.127422 & 5.37192 & 790.5694 & 0.00000 \\
\hline \multirow{2}{*}{ Hero Honda } & Return & 0.000758 & 0.02440 & 0.368415 & 6.74474 & 2340.882 & 0.00000 \\
\hline & Volume & 11.67354 & 2.12878 & -1.19735 & 3.21988 & 929.6163 & 0.00000 \\
\hline \multirow{2}{*}{ Hindalco } & Return & $9.55 \mathrm{E}-05$ & 0.02733 & -0.10158 & 6.57224 & 2057.422 & 0.00000 \\
\hline & Volume & $1.44 \mathrm{E}+01$ & 1.43994 & -0.47194 & 2.69279 & 158.386 & 0.00000 \\
\hline \multirow[b]{2}{*}{ HUL } & Return & 0.000374 & 0.02062 & 0.232934 & 7.19165 & 2857.772 & 0.00000 \\
\hline & Volu & 13.96108 & 1.31 & -1.22372 & 4.05002 & 1139.836 & 0.00000 \\
\hline
\end{tabular}


Investigation of Causal Relationship between Stock Prices and Trading Volume using...

Table 1 (cont.): Descriptive Statistics

\begin{tabular}{|c|c|c|c|c|c|c|c|}
\hline Company & & Mean & Std. Dev. & Skewness & Kurtosis & $\begin{array}{c}\text { Jarque- } \\
\text { Bera } \\
\end{array}$ & $\begin{array}{c}\text { Probability } \\
\text { (J-B Test) }\end{array}$ \\
\hline \multirow{2}{*}{ ICICI Bank } & Return & 0.000865 & 0.03115 & 0.063539 & 6.77274 & 2275.8 & 0.00000 \\
\hline & Volume & 13.71558 & 1.65758 & -0.37531 & 2.27062 & 174.9935 & 0.00000 \\
\hline \multirow{2}{*}{ IDFC } & Return & 0.000372 & 0.03282 & 0.309985 & 7.03337 & 1280.157 & 0.00000 \\
\hline & Volume & 15.42751 & 0.76251 & -0.90942 & 4.95464 & 548.3283 & 0.00000 \\
\hline \multirow{2}{*}{$\begin{array}{l}\text { Indusind } \\
\text { Bank }\end{array}$} & Return & -0.00277 & 0.03711 & 0.999178 & 9.73320 & 466.5747 & 0.00000 \\
\hline & Volume & 11.95744 & 0.83672 & -0.49373 & 2.88373 & 2.471523 & 0.00000 \\
\hline \multirow{2}{*}{ Infosys Tech } & Return & 0.001072 & 0.02798 & -0.51296 & 11.5818 & 12005.03 & 0.00000 \\
\hline & Volume & 14.29085 & 0.94301 & -0.51183 & 4.25157 & 420.149 & 0.00000 \\
\hline \multirow{2}{*}{ ITC } & Return & 0.000723 & 0.02166 & 0.054766 & 6.04103 & 1489.687 & 0.00000 \\
\hline & Volume & 16.28578 & 0.99920 & 0.75991 & 4.87688 & 938.0669 & 0.00000 \\
\hline \multirow{2}{*}{ Jindal Steel } & Return & 0.001282 & 0.03400 & 0.019498 & 11.0662 & 9065.918 & 0.00000 \\
\hline & Volume & 11.78362 & 2.19214 & -0.04721 & 1.95420 & 153.6762 & 0.00000 \\
\hline \multirow{2}{*}{ JP Associates } & Return & 0.001006 & 0.03992 & 0.172964 & 7.44033 & 3132.469 & 0.00000 \\
\hline & Volume & 15.422 & 1.389 & -0.70896 & 2.91899 & 318.6112 & 0.00000 \\
\hline \multirow{2}{*}{$\begin{array}{l}\text { Kotak } \\
\text { Mahindra }\end{array}$} & Return & 0.000873 & 0.04124 & -4.20005 & 85.7210 & 1103252 & 0.00000 \\
\hline & Volume & 11.54982 & 2.11796 & -0.26040 & 1.84202 & 258.6798 & 0.00000 \\
\hline \multirow{2}{*}{$\begin{array}{l}\text { Larsen and } \\
\text { Toubro }\end{array}$} & Return & 0.000863 & 0.02703 & 0.303249 & 7.33204 & 3056.726 & 0.00000 \\
\hline & Volume & 14.7004 & 0.90414 & 0.286726 & 3.29346 & 66.30852 & 0.00000 \\
\hline \multirow{2}{*}{$\begin{array}{l}\text { Mahindra \& } \\
\text { Mahindra }\end{array}$} & Return & 0.000464 & 0.03063 & -2.66483 & 64.4623 & 611660.2 & 0.00000 \\
\hline & Volume & 13.49617 & 1.10044 & -0.68336 & 3.61397 & 360.874 & 0.00000 \\
\hline \multirow{2}{*}{ Maruti Suzuki } & Return & 0.000875 & 0.02377 & -0.00664 & 5.40630 & 592.8001 & 0.00000 \\
\hline & Volume & 13.72688 & 0.95220 & 0.395702 & 3.16788 & 67.03209 & 0.00000 \\
\hline \multirow{2}{*}{$\begin{array}{l}\text { S\&P CNX } \\
\text { Nifty }\end{array}$} & Return & 0.000445 & 0.01651 & -0.21516 & 9.65084 & 7123.691 & 0.00000 \\
\hline & Volume & 18.03868 & 0.83478 & -0.01972 & 2.35823 & 66.32009 & 0.00000 \\
\hline \multirow{2}{*}{ NTPC } & Return & 0.000267 & 0.02034 & 0.02717 & 7.44369 & 1720.666 & 0.00000 \\
\hline & Volume & 14.99851 & 0.80601 & -0.29533 & 4.63866 & 264.4737 & 0.00000 \\
\hline \multirow{2}{*}{ ONGC } & Return & 0.00051 & 0.02510 & 0.155092 & 7.44255 & 3141.798 & 0.00000 \\
\hline & Volume & 12.77434 & 2.23025 & -0.81586 & 2.37417 & 483.9627 & 0.00000 \\
\hline \multirow{2}{*}{ PNB } & Return & 0.001089 & 0.02767 & -0.12974 & 7.76432 & 2607.668 & 0.00000 \\
\hline & Volume & 13.36897 & 0.99056 & 0.017088 & 3.57124 & 37.52415 & 0.00000 \\
\hline \multirow{2}{*}{ Ranbaxy } & Return & 0.000355 & 0.02524 & -0.13605 & 8.53593 & 5246.819 & 0.00000 \\
\hline & Volume & 13.29218 & 1.43711 & -1.43481 & 5.30049 & 2310.865 & 0.00000 \\
\hline \multirow{2}{*}{$\begin{array}{l}\text { Reliance } \\
\text { Industries }\end{array}$} & Return & 1.000999 & 0.02484 & 0.430889 & 8.42022 & 5463.284 & 000 \\
\hline & Volume & 16.06654 & 0.8 & 198 & 3.24097 & 14.29165 & 0000 \\
\hline \multirow{2}{*}{ Reliance Infra } & Return & & & & & 8902 & 000 \\
\hline & Volume & 13.09 & 1.54479 & 15784 & 45 & 150.6911 & 0000 \\
\hline \multirow{2}{*}{ SBI } & Return & & & & & & 000 \\
\hline & Volume & & 0.92 & & & & 000 \\
\hline \multirow{2}{*}{ SESA GOA } & Return & & & & 5.44870 & & 0000 \\
\hline & Volume & 12.32814 & 2.36362 & -0.17696 & 2.06868 & 155.5925 & 0.00000 \\
\hline \multirow{2}{*}{ TATA POWER } & Return & 0.000542 & 0.02691 & -0.03303 & 8.98691 & 5760.984 & 0.00000 \\
\hline & Volume & 12.77548 & 1.40117 & -0.21837 & 2.91127 & 31.9288 & 0.00000 \\
\hline & Return & 0.000304 & 0.02940 & -0.19625 & 5.77837 & 1264.996 & 0.00000 \\
\hline TATA STEE & Volume & 14.78705 & 0.97375 & -0.64250 & 3.19000 & 270.8918 & 0.00000 \\
\hline & Return & 0.00071 & 0.02224 & 0.177376 & 7.59858 & 1857.831 & 0.00000 \\
\hline TC & Volume & 14.38541 & 0.64344 & -0.59134 & 6.89886 & 1450.417 & 0.00000 \\
\hline & Return & 0.000852 & 0.02292 & 0.240956 & 5.89415 & 742.4712 & 0.00000 \\
\hline & Volume & 11.36563 & 0.97211 & -0.31671 & 4.35986 & 194.102 & 0.00000 \\
\hline
\end{tabular}

Source: Authors' Findings

EJBE 2014, 7 (14) 


\subsection{Unit Root Test}

In the initial stage of any time series analysis, the properties of the time series must be examined for the presence of stationarity. In the present paper, this task is accomplished by using two most widely used tests i.e. ADF (Augmented DickeyFuller) and PP (Phillips Perron). There are two motives behind running the stationarity test (i) confirmation of stability in the dataset; absence of which might show spurious results and (ii) determination of the maximum order of integration $\left(\rho_{\max }\right)$ to be added in the Vector Autoregressive (VAR) model for Toda and Yamamoto test. Table 2 reports the results of ADF and PP tests and it is apparent that securities and index' closing prices are integrated of order one while every security' trading volume data is stationary at level. Hence for each and every company, VAR model will add only one extra lag $\left(\rho_{\max }=1\right)$ for the execution of causality test.

Table 2: Unit Root Test

\begin{tabular}{|c|c|c|c|c|c|c|}
\hline \multirow{2}{*}{\multicolumn{2}{|c|}{ Company }} & \multicolumn{2}{|c|}{ Closing Prices } & \multicolumn{2}{|c|}{ Volume } & \multirow[b]{2}{*}{$\left(\rho_{\max }\right)$} \\
\hline & & \multirow{2}{*}{$\begin{array}{c}\begin{array}{c}\text { With } \\
\text { Intercept }\end{array} \\
-0.33305\end{array}$} & \multirow{2}{*}{$\begin{array}{c}\text { With } \\
\text { Intercept } \\
\text { and Trend }\end{array}$} & \multirow{2}{*}{$\begin{array}{c}\begin{array}{c}\text { With } \\
\text { Intercept }\end{array} \\
-4.61997\end{array}$} & \multirow{2}{*}{$\begin{array}{c}\text { With } \\
\text { Intercept } \\
\text { and Trend }\end{array}$} & \\
\hline \multirow{4}{*}{$A C C$} & I [0] (ADF) & & & & & \multirow{4}{*}{1} \\
\hline & I [1] (ADF) & -10.23022 & -10.27504 & - & - & \\
\hline & I [0] (PP) & -0.55117 & -2.69165 & -32.92096 & -37.87977 & \\
\hline & I [1] (PP) & -63.47590 & -63.47576 & - & - & \\
\hline \multirow{4}{*}{$\begin{array}{l}\text { Asian } \\
\text { Paints }\end{array}$} & I [0] (ADF) & 0.72465 & 1.34448 & -3.75620 & -6.66207 & \multirow{4}{*}{1} \\
\hline & I [1] (ADF) & -9.08954 & -9.81023 & - & - & \\
\hline & I [0] (PP) & 0.24674 & 1.45856 & -56.75569 & -61.59747 & \\
\hline & I [1] (PP) & -65.33445 & -65.83626 & - & - & \\
\hline \multirow{4}{*}{ Axis Bank } & I [0] (ADF) & -0.27759 & -2.64472 & -2.57631 & -5.81136 & \multirow{4}{*}{1} \\
\hline & I [1] (ADF) & -16.56492 & -16.58408 & - & - & \\
\hline & $\mathrm{I}[0]$ (PP) & -0.34787 & -2.77378 & -11.92045 & -37.20424 & \\
\hline & I [1] (PP) & -57.41353 & -57.41772 & - & - & \\
\hline \multirow{4}{*}{$\begin{array}{l}\text { Bank of } \\
\text { Baroda }\end{array}$} & I [0] (ADF) & -0.81480 & -2.41119 & -3.69692 & -4.17558 & \multirow{4}{*}{1} \\
\hline & I [1] (ADF) & -29.88766 & -29.89156 & - & - & \\
\hline & $\mathrm{I}[0]$ (PP) & -0.74262 & -2.27775 & -25.35390 & -29.99494 & \\
\hline & I [1] (PP) & -58.87344 & -58.86812 & - & - & \\
\hline \multirow{4}{*}{$\begin{array}{l}\text { BHARAT } \\
\text { PETROLEU } \\
\text { M }\end{array}$} & I [0] (ADF) & -1.67432 & -2.67606 & -3.86133 & -4.68252 & \multirow{4}{*}{1} \\
\hline & I [1] (ADF) & -20.81437 & -20.81678 & - & - & \\
\hline & I [0] (PP) & -2.10236 & -2.80664 & -28.33864 & -37.58443 & \\
\hline & I [1] (PP) & -62.74554 & -62.73898 & - & - & \\
\hline \multirow{4}{*}{$\begin{array}{l}\text { BHARTI } \\
\text { AIRTEL }\end{array}$} & I [0] (ADF) & -1.59027 & -1.31989 & -3.12067 & -4.05629 & \multirow{4}{*}{1} \\
\hline & I [1] (ADF) & -12.33739 & -12.37615 & - & - & \\
\hline & I [0] (PP) & -1.60130 & -1.51458 & -21.95059 & -33.64555 & \\
\hline & I [1] (PP) & -54.64790 & -54.68406 & - & - & \\
\hline \multirow{4}{*}{ BHEL } & I [0] (ADF) & -1.18464 & -1.13720 & -3.86836 & -5.11915 & \multirow{4}{*}{1} \\
\hline & I [1] (ADF) & -13.16960 & -13.18436 & - & - & \\
\hline & I [0] (PP) & -1.15994 & -1.16935 & -29.58349 & -36.54668 & \\
\hline & I [1] (PP) & -56.26061 & -56.26268 & - & - & \\
\hline
\end{tabular}


Investigation of Causal Relationship between Stock Prices and Trading Volume using...

Table 2 (cont.): Unit Root Test

\begin{tabular}{|c|c|c|c|c|c|c|}
\hline & \multicolumn{2}{|c|}{ Closing Prices } & \multicolumn{2}{|c|}{ Volume } & \multirow[b]{2}{*}{$\left(\rho_{\max }\right)$} \\
\hline Company & & \multirow{2}{*}{$\begin{array}{c}\begin{array}{c}\text { With } \\
\text { Intercept }\end{array} \\
-0.43857\end{array}$} & \multirow[t]{2}{*}{$\begin{array}{c}\text { With } \\
\text { Intercept } \\
\text { and Trend }\end{array}$} & \multirow{2}{*}{$\begin{array}{c}\begin{array}{c}\text { With } \\
\text { Intercept }\end{array} \\
-4.10286\end{array}$} & \multirow[t]{2}{*}{$\begin{array}{c}\text { With } \\
\text { Intercept } \\
\text { and Trend }\end{array}$} & \\
\hline \multirow{4}{*}{ Cipla } & I [0] (ADF) & & & & & \multirow{4}{*}{1} \\
\hline & I [1] (ADF) & -11.95947 & -11.97556 & - & - & \\
\hline & $\mathrm{I}[0]$ (PP) & -0.40157 & -3.13159 & -37.62442 & -47.78385 & \\
\hline & I [1] (PP) & -66.56309 & -66.57018 & - & - & \\
\hline \multirow{4}{*}{ Dr. Reddy } & I [0] (ADF) & 1.15998 & -0.94708 & -5.44682 & -6.27224 & \multirow{4}{*}{1} \\
\hline & I [1] (ADF) & -31.31353 & -31.37708 & - & - & \\
\hline & I [0] (PP) & 1.20822 & -0.92876 & -43.91622 & -47.94033 & \\
\hline & I [1] (PP) & -67.86950 & -67.95019 & - & - & \\
\hline \multirow{4}{*}{ GAIL } & I [0] (ADF) & -0.96124 & -2.21642 & -2.60757 & -3.80453 & \multirow{4}{*}{1} \\
\hline & I [1] (ADF) & -31.51293 & -31.50911 & - & - & \\
\hline & I [0] (PP) & -0.93568 & -2.05984 & -14.60382 & -26.50465 & \\
\hline & I [1] (PP) & -66.62651 & -13.39218 & - & - & \\
\hline \multirow{4}{*}{ Grasim } & I [0] (ADF) & -0.68944 & -2.51564 & -5.23203 & -5.31927 & \multirow{4}{*}{1} \\
\hline & I [1] (ADF) & -28.09403 & -65.53994 & - & - & \\
\hline & $\mathrm{I}[0]$ (PP) & -0.88597 & -2.77568 & -37.75699 & -37.74555 & \\
\hline & I [1] (PP) & -66.19390 & -66.18745 & - & - & \\
\hline \multirow{4}{*}{$\begin{array}{l}\text { Gujarat } \\
\text { Ambuja }\end{array}$} & I [0] (ADF) & -1.93765 & -2.41699 & -4.61516 & -4.81059 & \multirow{4}{*}{1} \\
\hline & I [1] (ADF) & -38.48837 & -38.47257 & - & - & \\
\hline & $\mathrm{I}[0]$ (PP) & -1.93175 & -2.40513 & -38.48801 & -41.12649 & \\
\hline & I [1] (PP) & -38.57890 & -38.57741 & - & - & \\
\hline \multirow{4}{*}{ HCL Tech } & I [0] (ADF) & -0.65562 & -2.99169 & -4.86727 & -5.23047 & \multirow{4}{*}{1} \\
\hline & I [1] (ADF) & -29.30822 & -29.43763 & - & - & \\
\hline & I [0] (PP) & -0.32634 & -3.04114 & -46.36591 & -47.79201 & \\
\hline & I [1] (PP) & -50.80855 & -51.55076 & - & - & \\
\hline \multirow{4}{*}{$\begin{array}{l}\text { Hero } \\
\text { Honda }\end{array}$} & $\mathrm{I}[0]$ (ADF) & -0.47971 & -2.40032 & -2.79262 & -3.17523 & \multirow{4}{*}{1} \\
\hline & I [1] (ADF) & -30.37252 & -30.37474 & - & - & \\
\hline & $\mathrm{I}[0]$ (PP) & -0.54474 & -2.49801 & -12.24628 & -23.51247 & \\
\hline & I [1] (PP) & -65.42448 & -65.42131 & - & - & \\
\hline \multirow{4}{*}{ Hindalco } & I [0] (ADF) & -2.37212 & -2.78167 & -3.39529 & -6.73276 & \\
\hline & I [1] (ADF) & -39.28929 & -39.28868 & - & - & \\
\hline & I [0] (PP) & -2.38866 & -2.82505 & -23.55892 & -51.42953 & 1 \\
\hline & I [1] (PP) & -64.45790 & -64.45363 & - & - & \\
\hline & I [0] (ADF) & 0.24231 & -1.02414 & -2.75849 & -3.83713 & \\
\hline & I [1] (ADF) & -31.38803 & -31.41447 & - & - & \\
\hline HUL & $\mathrm{I}[0](\mathrm{PP})$ & 0.62437 & -0.64417 & -22.99144 & -36.27395 & 1 \\
\hline & I [1] (PP) & -66.35115 & -66.40286 & - & - & \\
\hline & I [0] (ADF) & -1.03168 & -3.14866 & -3.21961 & -4.52781 & \\
\hline & I [1] (ADF) & -30.61085 & -30.60969 & - & - & \\
\hline ICICI Bank & $\mathrm{I}[0]$ (PP) & -0.92975 & -2.99820 & -11.49388 & -34.69967 & 1 \\
\hline & I [1] (PP) & -55.97047 & -55.96485 & - & - & \\
\hline & I [0] (ADF) & -2.12126 & -2.19636 & -4.24280 & -4.61935 & \\
\hline IDC & I [1] (ADF) & -41.39331 & -41.38535 & - & - & 1 \\
\hline IDFC & $\mathrm{I}[0]$ (PP) & -2.03526 & -2.07685 & -27.94030 & -29.42369 & 1 \\
\hline & I [1] (PP) & -41.41520 & -41.40794 & - & - & \\
\hline
\end{tabular}


Sushil BAJAJ \& Vibha DUA

Table 2 (cont.): Unit Root Test

\begin{tabular}{|c|c|c|c|c|c|c|}
\hline & \multicolumn{2}{|c|}{ Closing Prices } & \multicolumn{2}{|c|}{ Volume } & \multirow[b]{2}{*}{$\left(\boldsymbol{\rho}_{\max }\right)$} \\
\hline Company & & \multirow{2}{*}{$\begin{array}{c}\begin{array}{c}\text { With } \\
\text { Intercept }\end{array} \\
2.89119\end{array}$} & \multirow[t]{2}{*}{$\begin{array}{c}\text { With } \\
\text { Intercept } \\
\text { and Trend }\end{array}$} & \multirow{2}{*}{$\begin{array}{c}\begin{array}{c}\text { With } \\
\text { Intercept }\end{array} \\
-4.98365\end{array}$} & \multirow[t]{2}{*}{$\begin{array}{c}\text { With } \\
\text { Intercept } \\
\text { and Trend } \\
-4.98747\end{array}$} & \\
\hline \multirow{4}{*}{$\begin{array}{l}\text { Indusind } \\
\text { Bank }\end{array}$} & I [0] (ADF) & & & & & \multirow{4}{*}{1} \\
\hline & I [1] (ADF) & -10.55558 & -10.98172 & - & - & \\
\hline & $\mathrm{I}[0]$ (PP) & 3.27100 & 0.99164 & -4.91060 & -4.90978 & \\
\hline & I [1] (PP) & -60.46323 & -60.70997 & - & - & \\
\hline \multirow{4}{*}{$\begin{array}{l}\text { InfosysTec } \\
\mathrm{h}\end{array}$} & I [0] (ADF) & -1.05993 & -2.91478 & -3.93537 & -4.29098 & \multirow{4}{*}{1} \\
\hline & I [1] (ADF) & -64.78699 & -64.77975 & - & - & \\
\hline & I [0] (PP) & -1.00471 & -2.81604 & -34.41929 & -34.91416 & \\
\hline & I [1] (PP) & -64.83617 & -64.82832 & - & - & \\
\hline \multirow{4}{*}{ ITC } & I [0] (ADF) & 1.41671 & 2.99933 & -3.41548 & -4.36533 & \multirow{4}{*}{1} \\
\hline & I [1] (ADF) & -17.25556 & -16.60040 & - & - & \\
\hline & I [0] (PP) & 1.37018 & 1.90249 & -36.11046 & -49.54922 & \\
\hline & I [1] (PP) & -70.01122 & -69.44946 & - & - & \\
\hline \multirow{4}{*}{ Jindal Steel } & I [0] (ADF) & -1.28368 & -1.80517 & -2.62181 & -4.98319 & \multirow{4}{*}{1} \\
\hline & I [1] (ADF) & -10.74001 & -10.74572 & - & - & \\
\hline & I [0] (PP) & -1.15657 & -1.45799 & -8.93256 & -24.19580 & \\
\hline & I [1] (PP) & -53.11038 & -53.10498 & - & - & \\
\hline \multirow{4}{*}{$\begin{array}{l}\text { JPAssociat } \\
\text { es }\end{array}$} & I [0] (ADF) & -2.23663 & -3.15425 & -3.13237 & -4.71248 & \multirow{4}{*}{1} \\
\hline & I [1] (ADF) & -10.94639 & -10.94621 & - & - & \\
\hline & I [0] (PP) & -2.00347 & -2.81038 & -19.21803 & -33.42578 & \\
\hline & I [1] (PP) & -63.37666 & -63.37008 & - & - & \\
\hline \multirow{4}{*}{$\begin{array}{l}\text { Kotak } \\
\text { Mahindra }\end{array}$} & $\mathrm{I}[0]$ (ADF) & -1.77499 & -3.26359 & -2.90396 & -4.41111 & \multirow{4}{*}{1} \\
\hline & I [1] (ADF) & -58.53411 & -58.52705 & - & - & \\
\hline & I [0] (PP) & -1.69189 & -3.14656 & -11.76395 & -36.09748 & \\
\hline & I [1] (PP) & -58.44669 & -58.43931 & - & - & \\
\hline \multirow{4}{*}{$\begin{array}{l}\text { Larsen and } \\
\text { Toubro }\end{array}$} & $\mathrm{I}[0]$ (ADF) & -1.00748 & -2.43811 & -3.44027 & -4.64434 & \multirow{4}{*}{1} \\
\hline & I [1] (ADF) & -59.77467 & -59.76923 & - & - & \\
\hline & $\mathrm{I}[0]$ (PP) & -0.92237 & -2.30949 & -32.47228 & -41.88337 & \\
\hline & I [1] (PP) & -59.57256 & -59.56594 & - & - & \\
\hline \multirow{4}{*}{$\begin{array}{l}\text { Mahindra } \\
\text { nd } \\
\text { Mahindra }\end{array}$} & I [0] (ADF) & -1.03885 & -2.76336 & -4.48606 & -5.23066 & \\
\hline & I [1] (ADF) & -64.37708 & -64.37460 & - & - & \\
\hline & $\mathrm{I}[0]$ (PP) & -1.07811 & -2.83393 & -29.81580 & -37.61761 & 1 \\
\hline & I [1] (PP) & -64.35353 & -64.35084 & - & - & \\
\hline & I [0] (ADF) & -1.42364 & -2.65140 & -2.72593 & -4.95716 & \\
\hline Maruti & I [1] (ADF) & -47.58588 & -47.57624 & - & - & \\
\hline Suzuki & I [0] (PP) & -1.45857 & -2.75581 & -23.24196 & -34.37161 & 1 \\
\hline & I [1] (PP) & -47.55987 & -47.55007 & - & - & \\
\hline & I [0] (ADF) & -0.48996 & -2.54846 & -3.39980 & -4.55787 & \\
\hline S\&P CNX & I [1] (ADF) & -14.16680 & -14.17896 & - & - & \\
\hline Nifty & I [0] (PP) & -0.35944 & -2.42938 & -15.60183 & -39.24566 & 1 \\
\hline & I [1] (PP) & -61.95980 & -61.95857 & - & - & \\
\hline & I [0] (ADF) & -2.21096 & -1.86367 & -4.04148 & -3.97930 & \\
\hline & I [1] (ADF) & -10.73653 & -10.80302 & - & - & 1 \\
\hline NTPC & $\mathrm{I}[0]$ (PP) & -2.35837 & -2.14000 & -33.19996 & -34.11296 & 1 \\
\hline & I [1] (PP) & -45.90858 & -45.96830 & - & - & \\
\hline
\end{tabular}


Investigation of Causal Relationship between Stock Prices and Trading Volume using...

Table 2 (cont.): Unit Root Test

\begin{tabular}{|c|c|c|c|c|c|c|}
\hline & \multicolumn{2}{|c|}{ Closing Prices } & \multicolumn{2}{|c|}{ Volume } & \multirow[b]{2}{*}{$\left(\rho_{\max }\right)$} \\
\hline Company & & \multirow{2}{*}{$\begin{array}{c}\begin{array}{c}\text { With } \\
\text { Intercept }\end{array} \\
-0.75480\end{array}$} & \multirow{2}{*}{$\begin{array}{c}\text { With } \\
\text { Intercept } \\
\text { and Trend }\end{array}$} & \multirow{2}{*}{$\begin{array}{c}\begin{array}{c}\text { With } \\
\text { Intercept }\end{array} \\
-2.77662\end{array}$} & \multirow{2}{*}{$\begin{array}{c}\text { With } \\
\text { Intercept } \\
\text { and Trend }\end{array}$} & \\
\hline \multirow{4}{*}{ ONGC } & $\mathrm{I}[0]$ (ADF) & & & & & \multirow{4}{*}{1} \\
\hline & I [1] (ADF) & -13.86177 & -13.86606 & - & - & \\
\hline & I [0] (PP) & -0.80467 & -3.81501 & -7.81874 & -25.58082 & \\
\hline & I [1] (PP) & -58.81082 & -58.80569 & - & - & \\
\hline \multirow{4}{*}{ PNB } & I [0] (ADF) & -1.69380 & -2.10722 & -3.76647 & -4.22621 & \multirow{4}{*}{1} \\
\hline & I [1] (ADF) & -48.67783 & -48.68016 & - & - & \\
\hline & I [0] (PP) & -1.65639 & -1.97695 & -24.12821 & -27.58924 & \\
\hline & I [1] (PP) & -48.61780 & -48.61959 & - & - & \\
\hline \multirow{4}{*}{ Ranbaxy } & I [0] (ADF) & -1.99929 & -2.61347 & -3.58342 & -3.61504 & \multirow{4}{*}{1} \\
\hline & I [1] (ADF) & -63.89288 & -63.89017 & - & - & \\
\hline & $\mathrm{I}[0](\mathrm{PP})$ & -2.07186 & -2.79318 & -22.26048 & -28.57323 & \\
\hline & I [1] (PP) & -64.10105 & -64.09655 & - & - & \\
\hline \multirow{4}{*}{$\begin{array}{l}\text { Reliance } \\
\text { Industries }\end{array}$} & I [0] (ADF) & -0.99010 & -1.91675 & -3.63391 & -6.59182 & \multirow{4}{*}{1} \\
\hline & I [1] (ADF) & -33.98784 & -33.98402 & - & - & \\
\hline & I [0] (PP) & -1.07236 & -2.08798 & -34.89450 & -52.42726 & \\
\hline & I [1] (PP) & -63.31765 & -63.30980 & - & - & \\
\hline \multirow{4}{*}{$\begin{array}{l}\text { Reliance } \\
\text { Infra }\end{array}$} & I [0] (ADF) & -2.39579 & -2.73665 & -3.14288 & -4.60125 & \multirow{4}{*}{1} \\
\hline & I [1] (ADF) & -15.54836 & -15.55438 & - & - & \\
\hline & I [0] (PP) & -2.29974 & -2.63339 & -18.45449 & -29.91883 & \\
\hline & I [1] (PP) & -62.68646 & -62.68165 & - & - & \\
\hline \multirow{4}{*}{ SBI } & I [0] (ADF) & -1.10019 & -2.82704 & -4.45012 & -4.45092 & \multirow{4}{*}{1} \\
\hline & I [1] (ADF) & -59.89210 & -59.88659 & - & - & \\
\hline & $\mathrm{I}[0](\mathrm{PP})$ & -1.04044 & -2.68218 & -27.36136 & -27.35942 & \\
\hline & I [1] (PP) & -59.57581 & -59.56692 & - & - & \\
\hline \multirow{4}{*}{ SESA GOA } & I [0] (ADF) & -1.18134 & -1.75285 & -3.05950 & -4.60044 & \multirow{4}{*}{1} \\
\hline & I [1] (ADF) & -11.84122 & -11.84134 & - & - & \\
\hline & $\mathrm{I}[0](\mathrm{PP})$ & -1.22601 & -1.83809 & -6.22703 & -21.01804 & \\
\hline & I [1] (PP) & -58.62433 & -58.61672 & - & - & \\
\hline \multirow{4}{*}{$\begin{array}{l}\text { TATA } \\
\text { POWER }\end{array}$} & I [0] (ADF) & -1.11317 & -2.52919 & -2.85224 & -3.85068 & \\
\hline & I [1] (ADF) & -17.65114 & -17.64905 & - & - & \\
\hline & I [0] (PP) & -1.13558 & -2.40494 & -19.99430 & -29.91434 & 1 \\
\hline & I [1] (PP) & -64.19872 & -64.19054 & - & - & \\
\hline & I [0] (ADF) & -1.65342 & -2.10720 & -3.55346 & -4.60167 & \\
\hline TATA & I [1] (ADF) & -31.26160 & -31.26047 & - & - & \\
\hline STEEL & I [0] (PP) & -1.77550 & -2.33723 & -24.64568 & -33.93580 & 1 \\
\hline & I [1] (PP) & -64.45103 & -64.44468 & - & - & \\
\hline & I [0] (ADF) & 0.11450 & -1.73487 & -4.96234 & -5.96205 & \\
\hline & I [1] (ADF) & -33.74925 & -33.76686 & - & - & \\
\hline ICS & $\mathrm{I}[0](\mathrm{PP})$ & 0.32152 & -1.50011 & -36.67851 & -36.87090 & 1 \\
\hline & I [1] (PP) & -46.12200 & -46.17809 & - & - & \\
\hline & I [0] (ADF) & -0.43948 & -1.64111 & -5.99773 & -8.01508 & \\
\hline ULTRATEC & I [1] (ADF) & -10.44074 & -10.46026 & - & - & \\
\hline $\mathrm{H}$ & $\mathrm{I}[0]$ (PP) & -0.09372 & -1.31694 & -41.01033 & -40.31200 & 1 \\
\hline & I [1] (PP) & -44.23274 & -44.23741 & - & - & \\
\hline
\end{tabular}

Source: Authors' Findings

ADF and PP tests P-Values: - [(With Intercept) @ 1\% (-3.43), @ 5\% (-2.86) and @ 10\% (-2.56)]

ADF and PP tests P-Values: - [(With Intercept \& Trend) @ 1\% (-3.96), @ 5\% (-3.41) and @ 10\% (-3.12)]

EJBE 2014, 7 (14)

Page | 167 


\subsection{Toda and Yamamoto Granger Causality Results}

Following the methodological approach described earlier, appropriate maximum lagged length $(k)$ for the variables in the VAR is chosen as suggested by two usual information criterions i.e. AIC and FPE. However, these criterions might show inappropriate results because it is not possible to find the best model with many datasets. To remove the possibility of any error, Lagrange multiplier (LM) test is also run for testing the hypothesis of "no residual serial correlation" and required adjustments are made in the lagged length to make the VAR model parsimonious. The results of Wald statistics along with their appropriate lag length are shown in Table 3. Results clearly depict that 26 per cent of the individual securities' (i.e. 10 securities) and an index have bilateral causality between price and trading volume series whereas around 36 per cent of the sample securities (i.e. 14 securities) have demonstrated unilateral causality from closing prices series to volume that can be interpreted as securities' price is the major factor impacting the investors' decision to go for trading (buying/selling). On the other hand, unilateral causality from trading volume to closing price has also been registered but only in case of 3 securities (i.e. 8 per cent of the sample size). Besides this 31 per cent of the sample size (i.e. 12 securities) has shown absence of cause and effect relationship between the volume and closing prices.

\subsection{Vector Autoregression (VAR) Results}

The results of Vector Autoregression (VAR) for the 39 individual securities and an index are displayed in Table 4 (a-g). Significant stock returns' coefficients at first lag in the volume equation evidence the leading role of stock returns in case of around 80 per cent (i.e. 31 securities) of the individual securities which explains the prominent role of stock return over trading volume i.e. investors' buying/selling decision largely depends upon the variation in stock returns. However, 13 per cent of the individual securities (i.e. 5 securities) and an index have shown the deterministic role of trading volume that can be interpreted as investors' buying/selling decision (demand and supply interaction) determines the direction (positive/negative) of stock returns and around 7 per cent (i.e. Reliance infra, NTPC and Tata Steel) didn't find lead-lag relation because both variables are impacting each other either up to the same number of lags or not impacting each other. Besides lead/lag relationship, VAR results can also be used to depict the presence and direction of causality between returns and trading volume that exhibit bilateral causality incase of 29 individual securities' and an index whereas 8 securities (i.e. ACC, Bank of Baroda, Gujarat Ambuja, Hero Honda, Hindalco, ICICl Bank, PNB and Cipla) have shown unilateral causality from stock returns to trading volume but not the vice versa and only one security i.e. Reliance industries has shown the direction of causality from trading volume to return. On the other hand, one security i.e. NTPC has shown the absence of interaction between stock returns and trading volume. 
Investigation of Causal Relationship between Stock Prices and Trading Volume using...

Table 3: Wald Statistics (Toda and Yamamoto Granger Causality Test)

\begin{tabular}{|c|c|c|c|c|c|}
\hline \multirow[t]{2}{*}{ Company } & \multirow[t]{2}{*}{ Lags } & \multicolumn{2}{|c|}{$\begin{array}{l}\text { Stock Price does not cause Trading } \\
\text { Volume }\end{array}$} & \multicolumn{2}{|c|}{$\begin{array}{c}\text { Trading Volume does not cause } \\
\text { Stock Price }\end{array}$} \\
\hline & & Wald Statistics & P-value & Wald Statistics & P-value \\
\hline $\mathrm{ACC}^{¥}$ & 3 & $10.37635^{* *}$ & 0.0156 & 1.726128 & 0.6311 \\
\hline Asian Paints $^{*}$ & 14 & $24.24841 * *$ & 0.0428 & 17.7869 & 0.2167 \\
\hline Axis Bank ${ }^{\wedge}$ & 6 & $45.04147^{*}$ & 0.0000 & $18.1514^{*}$ & 0.0059 \\
\hline Bank of Baroda $^{¥}$ & 4 & $43.90302^{*}$ & 0.0000 & 4.510166 & 0.3413 \\
\hline BHARTI AIRTEL & 3 & 5.843096 & 0.1195 & 4.763714 & 0.1899 \\
\hline BHARAT PETRO ${ }^{*}$ & 6 & $23.94724^{*}$ & 0.0005 & 5.470955 & 0.4850 \\
\hline $\mathrm{BHEL}^{*}$ & 6 & 7.222133 & 0.3008 & $13.8082^{* *}$ & 0.0319 \\
\hline Cipla $^{¥}$ & 18 & 57.80687* & 0.0000 & 11.16491 & 0.8872 \\
\hline Dr. Reddy & 3 & 4.678754 & 0.1969 & 0.763846 & 0.8581 \\
\hline GAIL $^{*}$ & 4 & 19.99396* & 0.0005 & 5.606036 & 0.2306 \\
\hline Grasim $^{*}$ & 6 & $24.77522^{*}$ & 0.0004 & 6.714148 & 0.3481 \\
\hline Gujarat Ambuja $^{*}$ & 4 & $16.20145^{*}$ & 0.0028 & 3.379472 & 0.4964 \\
\hline HCL Tech & 13 & 17.71824 & 0.1685 & 12.17618 & 0.5133 \\
\hline Hero Honda $^{¥}$ & 4 & $30.63701^{*}$ & 0.0000 & 2.891069 & 0.5762 \\
\hline Hindalco ${ }^{\wedge}$ & 4 & $13.06944 * *$ & 0.0109 & $9.101771^{* * *}$ & 0.0586 \\
\hline $\mathrm{HUL}^{¥}$ & 9 & 7.650568 & 0.5697 & $19.56849 * *$ & 0.0208 \\
\hline $\mathrm{ICICI}$ Bank ${ }^{\wedge}$ & 4 & $3801.261^{*}$ & 0.0000 & 23.91005* & 0.0001 \\
\hline IDFC & 5 & 3.292527 & 0.6550 & 5.763224 & 0.3299 \\
\hline Indusind Bank ${ }^{\wedge}$ & 6 & $27.45202^{*}$ & 0.0001 & $12.93627^{* *}$ & 0.0441 \\
\hline Infosys Tech ${ }^{\wedge}$ & 7 & $15.90745^{* *}$ & 0.0260 & $15.22665^{* *}$ & 0.0332 \\
\hline $\mathrm{ITC}^{¥}$ & 6 & 7.592327 & 0.2695 & $13.03128 * *$ & 0.0425 \\
\hline Jindal Steel $^{¥}$ & 9 & $23.68445^{*}$ & 0.0048 & 5.429394 & 0.7954 \\
\hline JP Associates ${ }^{\wedge}$ & 3 & $36.23929 *$ & 0.0000 & $18.60735^{*}$ & 0.0003 \\
\hline Kotak Mahindra $^{¥}$ & 5 & $25.58323^{*}$ & 0.0001 & 4.555101 & 0.4725 \\
\hline Larsen and Toubro & 4 & 2.96941 & 0.5630 & 5.954726 & 0.2026 \\
\hline$M \& M^{\wedge}$ & 4 & $15.89515^{*}$ & 0.0032 & 19.70844* & 0.0006 \\
\hline Maruti Suzuki & 7 & 6.224529 & 0.5138 & 9.945861 & 0.1917 \\
\hline S\&P CNX Nifty & 7 & $16.96927^{* *}$ & 0.0176 & $16.84263^{* *}$ & 0.0184 \\
\hline NTPC & 10 & 4.006959 & 0.9470 & 6.368027 & 0.7835 \\
\hline ONGC $^{\wedge}$ & 4 & $48.6506^{*}$ & 0.0000 & 15.8905* & 0.0032 \\
\hline PNB & 4 & 6.196314 & 0.1850 & 3.093524 & 0.5423 \\
\hline Ranbaxy & 9 & 10.50263 & 0.3113 & 10.23135 & 0.3321 \\
\hline Reliance Industries & 3 & 1.559384 & 0.6686 & 5.647862 & 0.1301 \\
\hline Reliance Infra & 7 & 7.522181 & 0.3766 & 7.374879 & 0.3909 \\
\hline $\mathrm{SBI}^{*}$ & 9 & $17.36138 * *$ & 0.0433 & 6.484657 & 0.6906 \\
\hline SESA GOA & 4 & $52.0152 *$ & 0.0000 & $37.78407^{*}$ & 0.0000 \\
\hline TATA POWER $^{\wedge}$ & 8 & $18.61591^{* *}$ & 0.0171 & $16.30467 * *$ & 0.0382 \\
\hline TATA STEEL ${ }^{*}$ & 7 & $15.1773^{* *}$ & 0.0338 & 10.42101 & 0.1659 \\
\hline TCS & 6 & 9.684918 & 0.1386 & 7.147198 & 0.3074 \\
\hline ULTRATECH $^{*}$ & 6 & $33.20262 *$ & 0.0000 & 6.204568 & 0.4007 \\
\hline
\end{tabular}

Source: Authors' Findings

Note: $-\left({ }^{*}\right)\left[{ }^{* *}\right]\{* * *\}$ testify that values are significant @ (1\%), [5\%] and $\{10 \%\}$ level of significance. $\left({ }^{\wedge}\right)$ testifies the presence of bilateral causality between stock prices and trading volume. (¥) testifies the presence of unilateral causality between stock prices and trading volume. 
Table 4a: Vector Autoregression Model Results

\begin{tabular}{|c|c|c|c|c|c|c|c|c|c|c|}
\hline & \multicolumn{2}{|c|}{ ACC } & \multicolumn{2}{|c|}{ Bharti Airtel } & \multicolumn{2}{|c|}{ Dr. Reddy } & \multicolumn{2}{|c|}{ JP Associates } & \multicolumn{2}{|c|}{ Reliance Industries } \\
\hline & RETURN & VOLUME & RETURN & VOLUME & RETURN & VOLUME & RETURN & VOLUME & RETURN & VOLUME \\
\hline \multirow[t]{2}{*}{ C } & -0.001728 & $1.277452^{*}$ & $0.013514^{* *}$ & $1.263407^{*}$ & $0.01458^{* *}$ & $2.108566^{*}$ & 0.011837 & $0.988641^{*}$ & $0.966451^{*}$ & $2.100871^{*}$ \\
\hline & {$[-0.29526]$} & [ 10.4797] & [ 2.05476] & [ 8.57369] & [ 2.48119] & [ 14.2007] & [ 1.56684] & [ 8.82290] & [ 37.1014] & [ 4.33897] \\
\hline \multirow[t]{2}{*}{ RETURN(-1) } & $0.038803^{* *}$ & 2.858943* & -0.019226 & $0.831633^{* * *}$ & 0.006893 & 2.976251* & $0.082376^{*}$ & 2.777091* & $0.054302 *$ & 0.109558 \\
\hline & [ 2.40710] & [ 8.51714] & {$[-1.00826]$} & [-1.94650] & [ 0.42768$]$ & [ 7.30808] & [ 5.06021] & [ 11.5016] & [ 3.57003] & [ 0.38750$]$ \\
\hline \multirow[t]{2}{*}{ RETURN(-2) } & $-0.04311^{*}$ & $0.592695^{* * *}$ & $-0.072725^{*}$ & 0.037066 & $-0.034054^{* *}$ & 0.590327 & -0.01656 & $1.03527 *$ & $-0.025478^{* *}$ & -0.20614 \\
\hline & {$[-2.65162]$} & [ 1.75078] & {$[-3.82696]$} & [ 0.08705$]$ & {$[-2.10011]$} & [ 1.44073] & [-0.99845] & [ 4.20936] & {$[-1.67365]$} & {$[-0.72851]$} \\
\hline \multirow[t]{2}{*}{ RETURN(-3) } & 0.006436 & -0.059095 & -0.029443 & $-1.30372 *$ & -0.007142 & 0.296256 & 0.007366 & -0.276092 & -0.011496 & -0.393242 \\
\hline & [ 0.39601$]$ & {$[-0.17461]$} & [-1.54995] & {$[-3.06301]$} & {$[-0.44052]$} & [ 0.72314] & [0.44780] & {$[-1.13167]$} & {$[-0.75606]$} & {$[-1.39143]$} \\
\hline \multirow[t]{2}{*}{ VOLUME(-1) } & 0.001044 & $0.455989 *$ & 0.00103 & $0.538319 *$ & $-0 . c$ & $0.479373 *$ & -0.00014 & $0.526038^{*}$ & $0.001872^{* *}$ & $0.490798 *$ \\
\hline & [ 1.38362$]$ & [ 29.0115] & [ 1.24028$]$ & [ 28.9293] & {$[-0.35791]$} & [ 30.2252] & {$[-0.13061]$} & [ 33.1618] & [ 2.34073] & [ 33.0243] \\
\hline \multirow[t]{2}{*}{ VOLUME(-2) } & -0.000457 & $0.228873^{*}$ & 0.000119 & $0.153176^{*}$ & 0.000181 & 0.1706 & 0.001554 & 0.1993 & -0.00007 & $0.197492^{*}$ \\
\hline & {$[-0.56092]$} & [ 13.4941] & [ 0.12714$]$ & [ 7.28354] & [ 0.26174$]$ & [ 9.79221] & [ 1.29972] & [ 11.2368] & {$[-0.08069]$} & [ 12.0782] \\
\hline \multirow[t]{2}{*}{ VOLUME(-3) } & -0.000414 & $0.219922^{*}$ & $-0.002008^{* *}$ & $0.221543^{*}$ & $-0.00106 * * *$ & $0.179747^{*}$ & $-0.00212^{* *}$ & $0.210442^{*}$ & -0.00073 & $0.211453^{*}$ \\
\hline & {$[-0.54990]$} & [ 14.0363] & {$[-2.41795]$} & [ 11.9052] & [-1.69568] & [ 11.3615] & [-1.99032] & [ 13.3080] & {$[-0.91340]$} & [ 14.2287] \\
\hline F-Values & 2.260 .321 & 1.704 .059 & 436.737 & 1.376.176 & 189.854 & 8.342 .732 & 5.304 .617 & 2.854 .044 & 4.378 .562 & 1.748 .982 \\
\hline
\end{tabular}

\section{Source: Authors' Findings}

Note: - $\left.{ }^{*}\right)$ testifies that values are significant at 1 per cent level (critical value: 2.58$) ;\left({ }^{* *}\right)$ testifies that values are significant at 5 per cent level

(critical value: 1.96$) ;(* * *)$ testifies that values are significant at 10 per cent level of significance (critical value 1.65) 


\begin{tabular}{|c|c|c|c|c|c|c|c|c|c|c|}
\hline & \multicolumn{2}{|c|}{ Bank of Baroda } & \multicolumn{2}{|c|}{ GAIL } & \multicolumn{2}{|c|}{ Gujarat Ambuja } & \multicolumn{2}{|c|}{ Hero Honda } & \multicolumn{2}{|c|}{ Hindalco } \\
\hline & RETURN & VOLUME & RETURN & VOLUME & RETURN & VoLUME & RETURN & VOLUME & RETURN & VOLUME \\
\hline c & -0.003471 & $0.908451^{*}$ & -0.001924 & $0.445772^{*}$ & -0.005938 & $1.578406 *$ & 0.000935 & $0.314759^{*}$ & 0.000314 & $0.768444^{*}$ \\
\hline & {$[-0.67469]$} & [ 8.56473] & {$[-0.68395]$} & [ 5.75521] & {$[-0.97485]$} & [ 10.4097] & [ 0.41651] & [ 5.04290] & [0.06730] & [ 7.14795] \\
\hline RETURN(-1) & $0.099714^{*}$ & $2.813667^{*}$ & 0.002569 & $2.247389 *$ & 0.000169 & $3.000706^{*}$ & $0.049921^{*}$ & $2.539546^{*}$ & $0.089207^{*}$ & 1.608267* \\
\hline & [ 6.17873] & [ 8.45606] & [ 0.15917] & [ 5.05639] & [0.01046] & [ 7.45390] & [ 3.09496] & [ 5.66332] & [ 5.52812] & [ 4.32080] \\
\hline RETURN(-2) & -0.025506 & $1.682172^{*}$ & $-0.044504 *$ & $1.692313^{*}$ & $-0.055255^{*}$ & $1.272152^{*}$ & $-0.05739^{*}$ & $0.835093^{* * *}$ & 0.007549 & $0.879317^{* *}$ \\
\hline & [-1.55985] & [ 4.98966] & [-2.74893] & [ 3.79555] & {$[-3.39248]$} & [ 3.13781] & {$[-3.54794]$} & [ 1.85718$]$ & [ 0.46538] & [ 2.35002] \\
\hline RETURN(-3) & -0.015998 & -0.27779 & -0.003636 & 0.577203 & 0.00003 & $-0.71087^{* * *}$ & $-0.07251^{*}$ & $-1.496342^{*}$ & $-0.04607^{*}$ & -0.055227 \\
\hline & {$[-0.97598]$} & {$[-0.82197]$} & {$[-0.22435]$} & [1.29314] & [0.00198] & {$[-1.75097]$} & {$[-4.48039]$} & {$[-3.32564]$} & {$[-2.83963]$} & {$[-0.14759]$} \\
\hline RETURN(-4) & -0.021464 & $-0.871507^{* *}$ & 0.008244 & 0.083634 & 0.014207 & -0.556175 & -0.00961 & $-1.538193^{*}$ & -0.01017 & $-0.752651^{* *}$ \\
\hline & {$[-1.31789]$} & {$[-2.59536]$} & [ 0.50928] & {$[0.18760]$} & [0.87164] & {$[-1.37088]$} & {$[-0.59222]$} & {$[-3.41134]$} & {$[-0.62943]$} & [-2.01992] \\
\hline VOLUME(-1) & 0.000303 & $0.510804^{*}$ & -0.000111 & $0.422269^{*}$ & 0.0002 & $0.407419^{*}$ & 0.00006 & $0.443259^{*}$ & 0.000287 & $0.422186^{*}$ \\
\hline & [ 0.39359] & [ 32.1391] & {$[-0.19148]$} & [26.5150] & [0.31111] & [ 25.4141] & {$[0.10287]$} & [ 27.9090] & [ 0.41643] & [ 26.5172] \\
\hline VOLUME(-2) & 0.000226 & $0.138753^{*}$ & -0.000522 & $0.20592^{*}$ & 0.000476 & $0.205698^{*}$ & 0.000472 & $0.216211^{*}$ & 0.000159 & $0.217158^{*}$ \\
\hline & [ 0.26177] & [ 7.79438] & {$[-0.84173]$} & [ 12.0548] & [0.69042] & [ 11.9842] & [0.76207] & [ 12.5435] & [0.21427] & [ 12.6602] \\
\hline VOLUME(-3) & -0.00009 & $0.116979 *$ & $0.001219 * *$ & $0.177547^{*}$ & 0.000302 & $0.146518^{*}$ & -0.00023 & $0.151257^{*}$ & 0.000268 & $0.149062^{*}$ \\
\hline & {$[-0.10304]$} & [ 6.58811] & [ 1.96605] & [ 10.3946] & {$[0.43817]$} & [ 8.53929] & {$[-0.36999]$} & [ 8.77300] & [0.36030] & [ 8.69260] \\
\hline VOLUME(-4) & -0.000134 & $0.162282^{*}$ & -0.000412 & $0.159946 *$ & -0.000523 & $0.130627^{*}$ & -0.00031 & $0.162481^{*}$ & -0.00073 & $0.158235^{*}$ \\
\hline & {$[-0.17537]$} & [ 10.2779] & {$[-0.71279]$} & [ 10.0516] & {$[-0.81655]$} & [ 8.19899] & {$[-0.54606]$} & [ 10.2695] & {$[-1.06025]$} & [ 9.96303] \\
\hline F-Values & 5.434.045 & 1.646 .514 & 1.587 .804 & 3.383.359 & 1.885 .944 & 9.319 .657 & 5.701 .048 & 4295.27 & 5.152 .705 & $\begin{array}{l}2.042 .428 \\
\end{array}$ \\
\hline
\end{tabular}


Table 4c: Vector Autoregression Model Results

Table 4c: Vector Autoregression Model Results

\begin{tabular}{|c|c|c|c|c|c|c|c|c|c|c|}
\hline & \multicolumn{2}{|c|}{ ICICI BANK } & \multicolumn{2}{|c|}{ Larsen \& Toubro } & \multicolumn{2}{|c|}{ Mahindra \& Mahindra } & \multicolumn{2}{|c|}{ ONGC } & \multicolumn{2}{|c|}{ PNB } \\
\hline & RETURN & VOLUME & RETURN & VOLUME & RETURN & VOLUME & RETURN & VOLUME & RETURN & VOLUME \\
\hline \multirow[t]{2}{*}{ c } & 0.003009 & $0.481285^{*}$ & 0.007079 & $1.398381^{*}$ & -0.008156 & $1.161322^{*}$ & -0.00088 & $0.281571^{*}$ & -0.010467 & $1.191055^{*}$ \\
\hline & [ 0.69523] & [ 5.92678] & [ 0.90904] & [ 9.71360$]$ & {$[-1.23511]$} & [ 9.45388] & {$[-0.36315]$} & [ 4.64411] & {$[-1.33054]$} & [ 7.87175$]$ \\
\hline \multirow[t]{2}{*}{ RETURN(-1) } & $0.105152 *$ & $1.915685^{*}$ & $0.074933^{*}$ & $2.334378^{*}$ & $0.112808^{*}$ & $2.338871^{*}$ & $0.066395^{*}$ & $1.88395^{*}$ & $0.045545^{* *}$ & $0.882889 * *$ \\
\hline & [ 6.49797] & [ 6.30911] & [ 4.63551] & [ 7.81186] & [ 6.98727] & [ 7.78784] & [ 4.08027] & [ 4.61438] & [ 2.35584] & [ 2.37438] \\
\hline \multirow[t]{2}{*}{ RETURN(-2) } & $-0.038236 * *$ & 0.255822 & $-0.04569 *$ & 0.081247 & $-0.03155^{* * *}$ & $0.525442^{* * *}$ & $-0.03846 * *$ & $0.714843^{* * *}$ & -0.015968 & 0.03734 \\
\hline & {$[-2.34068]$} & [ 0.83463$]$ & {$[-2.80298]$} & {$[0.26966]$} & {$[-1.92926]$} & [ 1.72701$]$ & {$[-2.35555]$} & [ 1.74520$]$ & {$[-0.82586]$} & [ 0.10041$]$ \\
\hline \multirow[t]{2}{*}{ RETURN(-3) } & 0.00007 & -0.381814 & $0.049494 *$ & -0.145147 & -0.017405 & $-0.723697^{* *}$ & 0.000741 & -0.647167 & $-0.03216^{* * *}$ & -0.233317 \\
\hline & [ 0.00418] & {$[-1.24599]$} & [ 3.03626$]$ & {$[-0.48167]$} & {$[-1.06355]$} & {$[-2.37731]$} & [ 0.04554] & {$[-1.58411]$} & {$[-1.66571]$} & {$[-0.62827]$} \\
\hline \multirow[t]{2}{*}{ RETURN(-4) } & -0.023426 & -0.359169 & 0.00921 & -0.428949 & -0.005052 & $-0.56494 * * *$ & 0.016404 & $0.789229 * * *$ & 0.009608 & -0.171791 \\
\hline & {$[-1.44378]$} & {$[-1.17972]$} & [ 0.56660$]$ & {$[-1.42759]$} & {$[-0.31096]$} & {$[-1.86924]$} & [ 1.01036$]$ & [ 1.93739] & [ 0.50058$]$ & {$[-0.46537]$} \\
\hline \multirow[t]{2}{*}{ VOLUME(-1) } & 0.000653 & $0.543984^{*}$ & -0.00047 & $0.4730^{*}$ & 0.000106 & $0.481377^{*}$ & $0.00238^{*}$ & $0.472313^{*}$ & -0.000216 & $0.495057^{*}$ \\
\hline & [ 0.77017] & [ 34.1836] & {$[-0.54515]$} & [ 29.5262] & [ 0.12284$]$ & [ 30.1273] & [ 3.71679] & [ 29.3978] & {$[-0.21818]$} & [ 26.0222] \\
\hline \multirow[t]{2}{*}{ VOLUME(-2) } & 0.00021 & $0.1588^{*}$ & $0.002363^{* *}$ & $0.164982 *$ & $0.00181^{* * *}$ & $0.174964 *$ & -0.00109 & $0.190504^{*}$ & 0.001634 & $0.14654 *$ \\
\hline & [ 0.21772] & [ 8.76099] & [ 2.47761] & [ 9.35838] & [ 1.90575$]$ & [ 9.90181] & {$[-1.55067]$} & [ 10.8241] & [ 1.48112] & [ 6.90669] \\
\hline \multirow[t]{2}{*}{ VOLUME(-3) } & -0.000614 & $0.081674 *$ & -0.0004 & $0.131377^{*}$ & 0.000308 & $0.113393^{*}$ & -0.00054 & $0.157156^{*}$ & -0.000937 & $0.093146^{*}$ \\
\hline & {$[-0.63584]$} & [ 4.50532] & {$[-0.42014]$} & [ 7.44617] & [ 0.32424$]$ & [ 6.41415] & {$[-0.77550]$} & [ 8.92750] & {$[-0.85012]$} & [ 4.39214] \\
\hline \multirow[t]{2}{*}{ VOLUME(-4) } & -0.000408 & $0.180461^{*}$ & $-0.00192^{* *}$ & $0.135394 *$ & $-0.00158^{* * *}$ & $0.144292^{*}$ & -0.00064 & $0.158116^{*}$ & 0.00038 & $0.176116^{*}$ \\
\hline & {$[-0.48190]$} & [ 11.3636] & {$[-2.21901]$} & [ 8.47363] & {$[-1.86151]$} & [ 9.09556 ] & {$[-1.00176]$} & [ 9.84298] & [ 0.38561$]$ & [ 9.28983] \\
\hline F-Values & 6.152 .269 & 3.409 .274 & 6.122 .636 & 1.106 .679 & 7.504 .632 & 1.327 .325 & 4.627 .757 & 5.512 .638 & 1.635 .338 & 8.520 .377 \\
\hline
\end{tabular}


Table 4d: Vector Autoregression Model Results

\begin{tabular}{|c|c|c|c|c|c|c|c|c|c|c|}
\hline & \multicolumn{2}{|c|}{ Sesa Goa } & \multicolumn{2}{|c|}{ IDFC } & \multicolumn{2}{|c|}{ Kotak Mahindra } & \multicolumn{2}{|c|}{ Axis Bank } & \multicolumn{2}{|c|}{ Bharat Petroleum } \\
\hline & RETURN & VOLUME & RETURN & VOLUME & RETURN & VOLUME & RETURN & VOLUME & RETURN & VOLUME \\
\hline \multirow[t]{2}{*}{$C$} & -0.001502 & $0.252287^{*}$ & -0.006661 & $2.767222^{*}$ & 0.00210 & $0.307044 *$ & 0.005861 & $0.330915^{*}$ & -0.007343 & $0.704821 *$ \\
\hline & {$[-0.48943]$} & [ 4.45935] & {$[-0.35004]$} & [ 8.78848] & [0.55395] & [ 4.77939] & [ 1.43441] & [ 4.42183] & {$[-0.81872]$} & [ 6.34364] \\
\hline \multirow[t]{2}{*}{ RETURN(-1) } & $0.072013^{*}$ & $1.594722^{*}$ & 0.02288 & $1.22022^{*}$ & $0.09040^{*}$ & 1.980981* & 0.02339 & $3.286887 *$ & 0.006763 & $0.656779 *$ \\
\hline & [ 4.32844] & [ 5.19976] & {$[0.97781]$} & [ 3.15163] & [ 5.58131] & [ 7.29663] & [ 1.40222] & [ 10.7589] & [ 0.41453] & [3.24955] \\
\hline \multirow[t]{2}{*}{ RETURN(-2) } & $-0.03170 * * *$ & 0.492807 & $-0.06743^{*}$ & 0.419611 & 0.00340 & 1.017933* & $-0.043963 * *$ & $1.740177^{*}$ & 0.002323 & 0.066993 \\
\hline & {$[-1.90178]$} & [ 1.60339] & {$[-2.87563]$} & [ 1.08149] & [ 0.20688] & [ 3.70731] & {$[-2.59382]$} & [ 5.60595] & [ 0.14223] & [0.33115] \\
\hline \multirow[t]{2}{*}{ RETURN(-3) } & -0.008069 & $-1.319797^{*}$ & -0.01433 & -0.179125 & $0.0305^{* * *}$ & 0.069341 & -0.022821 & $0.546918^{* * *}$ & $0.041664 * * *$ & $-0.622237^{*}$ \\
\hline & {$[-0.48847]$} & {$[-4.33437]$} & {$[-0.60976]$} & {$[-0.46065]$} & [ 1.86167] & [ 0.25232] & {$[-1.34395]$} & [ 1.75859] & [ 2.55168] & {$[-3.07618]$} \\
\hline \multirow[t]{2}{*}{ RETURN(-4) } & 0.02541 & -0.48417 & $-0.04089 * * *$ & -0.438656 & 0.00180 & 0.129685 & -0.017494 & 0.222668 & 0.011128 & 0.241127 \\
\hline & [ 1.55085] & {$[-1.60300]$} & {$[-1.74356]$} & {$[-1.13042]$} & [ 0.10879] & [ 0.47202] & {$[-1.03005]$} & [0.71585] & [ 0.68070] & [ 1.19062] \\
\hline \multirow[t]{2}{*}{ RETURN(-5) } & - & - & -0.014561 & -0.151106 & -0.00580 & -0.390398 & -0.007223 & -0.153067 & -0.022283 & -0.195285 \\
\hline & - & - & {$[-0.62127]$} & {$[-0.38964]$} & {$[-0.35261]$} & [-1.42765] & {$[-0.42810]$} & {$[-0.49537]$} & [-1.36435] & {$[-0.96520]$} \\
\hline \multirow[t]{2}{*}{ RETURN(-6) } & - & - & - & - & - & - & $-0.044059^{* *}$ & $-0.56235^{* * *}$ & -0.003361 & -0.155168 \\
\hline & - & - & - & - & - & - & {$[-2.62043]$} & {$[-1.82621]$} & {$[-0.20594]$} & {$[-0.76740]$} \\
\hline \multirow[t]{2}{*}{ VOLUME(-1) } & $0.005129 *$ & $0.567219^{*}$ & -0.001451 & $0.464422^{*}$ & 0.00010 & 0.47998* & $0.002155^{* *}$ & $0.470754^{*}$ & 0.001662 & $0.397465^{*}$ \\
\hline & [ 5.75121] & [ 34.5009] & {$[-1.03233]$} & [ 19.9688] & {$[0.05310]$} & [ 29.8771] & [2.36575] & [ 28.2178] & [ 1.26231] & [ 24.3612] \\
\hline \multirow[t]{2}{*}{ VOLUME(-2) } & $-0.002845^{*}$ & $0.152024 *$ & 0.001234 & $0.102082^{*}$ & -0.0010 & $0.181185^{*}$ & $-0.002477^{* *}$ & $0.150092^{*}$ & $-0.00250 * * *$ & $0.141816^{*}$ \\
\hline & {$[-2.79554]$} & [8.10282] & [0.79589] & [ 3.97941] & {$[-0.91365]$} & [ 10.1824] & {$[-2.47320]$} & [ 8.18299] & {$[-1.78719]$} & [ 8.16471] \\
\hline \multirow[t]{2}{*}{ VOLUME(-3) } & -0.001398 & $0.097156^{*}$ & -0.001404 & $0.099724^{*}$ & -0.0009 & $0.101743^{*}$ & $0.00238^{* *}$ & $0.084706^{*}$ & 0.000575 & $0.108961 *$ \\
\hline & {$[-1.37265]$} & [ 5.17468] & {$[-0.90521]$} & [3.88622] & {$[-0.80427]$} & [ 5.66859] & [ 2.36343] & [ 4.59281] & [0.40881] & [ 6.24847] \\
\hline \multirow[t]{2}{*}{ VOLUME(-4) } & -0.000703 & $0.163256^{*}$ & -0.000666 & $0.044888^{* * *}$ & -0.00050 & $0.086273^{*}$ & -0.001379 & $0.103568 *$ & 0.001443 & $0.109156^{*}$ \\
\hline & {$[-0.78932]$} & [ 9.94692] & {$[-0.42962]$} & [ 1.75023] & {$[-0.46747]$} & [ 4.85360] & {$[-1.36904]$} & [ 5.61384] & [ 1.02389] & [ 6.25108] \\
\hline \multirow[t]{2}{*}{ VOLUME(-5) } & - & - & $0.002746 * *$ & $0.10956^{*}$ & $0.00220 * *$ & $0.124251^{*}$ & 0.000292 & $0.117569 *$ & -0.000363 & $0.117237^{*}$ \\
\hline & - & - & [ 1.96019] & [ 4.72652] & {$[2.25779]$} & {$[7.74860]$} & {$[0.29141]$} & [ 6.40490] & {$[-0.25817]$} & [ 6.73246] \\
\hline \multirow[t]{2}{*}{ VOLUME(-6) } & - & - & - & - & - & - & -0.001324 & $0.047064^{*}$ & -0.000207 & $0.069960^{*}$ \\
\hline & - & - & - & - & - & - & {$[-1.45607]$} & [ 2.82598] & {$[-0.15808]$} & [ 4.30671] \\
\hline F-Values & 8.369 .753 & 5.996 .783 & 1.880 .673 & 1.798 .872 & 42.164 & 3.205.257 & 2.630 .845 & 2.378 .758 & 1.158 .392 & 1.001 .369 \\
\hline
\end{tabular}


Table 4e: Vector Autoregression Model Results

\begin{tabular}{|c|c|c|c|c|c|c|c|c|c|c|}
\hline & \multicolumn{2}{|c|}{ BHEL } & \multicolumn{2}{|c|}{ Grasim } & \multicolumn{2}{|c|}{ Indusind Bank } & \multicolumn{2}{|c|}{ ITC } & \multicolumn{2}{|c|}{ TCS } \\
\hline & RETURN & VOLUME & RETURN & VOLUME & RETURN & VOLUME & RETURN & VOLUME & RETURN & VOLUME \\
\hline \multirow[t]{2}{*}{ C } & 0.00826 & 0.919597* & $-0.011898^{* *}$ & 1.095159* & 0.043122 & 347.618 & 0.00508 & $1.258013^{*}$ & -0.00778 & 3.551281* \\
\hline & [ 0.94084$]$ & [ 7.20047] & {$[-2.20462]$} & [ 8.66129] & [ 0.49401] & [ 1.52035$]$ & [ 0.78199] & [ 7.64934] & {$[-0.52229]$} & [ 10.0960] \\
\hline \multirow[t]{2}{*}{ RETURN(-1) } & $0.04126^{* *}$ & -0.042137 & $0.096263^{*}$ & 2.567 & 8601 & -1.370 .854 & -0.013945 & 2.397839* & 0.004252 & -1 \\
\hline & {$[2.53844]$} & {$[-0.17814]$} & [ 5.95955] & [ 6.78332] & {$[-0.38956]$} & {$[-0.34791]$} & {$[-0.86249]$} & [ 5.85808] & {$[0.19379]$} & {$[-2.27004]$} \\
\hline \multirow[t]{2}{*}{ RETURN(-2) } & $-0.0375^{* *}$ & -0.197961 & $-0.034696 * *$ & 1.294753* & 0.048301 & $7.306669 * *$ & $-0.02943^{* *}$ & 0.496554 & $-0.07727^{*}$ & 0.209161 \\
\hline & {$[-2.30258]$} & {$[-0.83623]$} & {$[-2.12851]$} & [ 3.39012] & [ 0.34572$]$ & [ 1.99661$]$ & {$[-1.81242]$} & [ 1.20783] & {$[-3.51775]$} & [ 0.40317] \\
\hline \multirow[t]{2}{*}{ RETURN(-3) } & -0.0248 & $-0.628385^{*}$ & $0.069023^{*}$ & 0.547817 & 0.22492 & $6.314425^{* * *}$ & -0.021082 & -0.2240 & $-0.07439 *$ & -0.407751 \\
\hline & {$[-1.52867]$} & {$[-2.65759]$} & [ 4.22836$]$ & [ 1.43233] & [ 1.59112] & [ 1.70534$]$ & {$[-1.29780]$} & {$[-0.54467]$} & {$[-3.38002]$} & {$[-0.78444]$} \\
\hline \multirow[t]{2}{*}{ RETURN(-4) } & -0.0002 & 0.003658 & 0.008674 & $-0.73861 * * *$ & 0.039209 & $-6.87907^{* * *}$ & 0.023148 & -0.291666 & $-0.04328^{* *}$ & -0.473923 \\
\hline & {$[-0.01203]$} & {$[0.01546]$} & [0.53166] & {$[-1.93219]$} & [ 0.28442$]$ & {$[-1.90507]$} & [ 1.42451$]$ & {$[-0.70897]$} & [-1.96647] & {$[-0.91169]$} \\
\hline \multirow[t]{2}{*}{ RETURN(-5) } & 0.00041 & -0.073755 & $0.040779 * *$ & 0.155989 & 0.217998 & $8.205986 * *$ & -0.001348 & -0.548454 & 0.002241 & -0.735831 \\
\hline & {$[0.02500]$} & {$[-0.31183]$} & [ 2.50145] & [ 0.40839] & [ 1.54600$]$ & [ 2.22173] & {$[-0.08297]$} & {$[-1.33335]$} & [ 0.10208$]$ & [-1.41924] \\
\hline \multirow[t]{2}{*}{ RETURN(-6) } & $-0.02880 * * *$ & -0.292823 & -0.02071 & $-0.897989 * *$ & -0.214174 & -3.064 .568 & -0.007601 & 0.661635 & -0.00575 & -0.347015 \\
\hline & {$[-1.77446]$} & [-1.23917] & [-1.27587] & {$[-2.36118]$} & {$[-1.46940]$} & {$[-0.80269]$} & {$[-0.46788]$} & [ 1.60870$]$ & {$[-0.26189]$} & {$[-0.6$} \\
\hline \multirow[t]{2}{*}{ VOLUME(-1) } & 0.00058 & $0.477232 *$ & 0.000598 & $0.419343^{*}$ & 0.004135 & $0.432312 *$ & -0.000793 & $0.40378^{*}$ & $0.001794^{* * *}$ & $0.361949 *$ \\
\hline & {$[0.52236]$} & [ 29.4586] & {$[0.87030]$} & [ 26.0302] & {$[0.71162]$} & [ 2.84029] & {$[-1.24552]$} & [ 25.0623] & [ 1.93277$]$ & [ 16.5108] \\
\hline \multirow[t]{2}{*}{ VOLUME(-2) } & 0.00003 & $0.091354^{*}$ & 0.00008 & $0.096187^{*}$ & 0.000115 & 0.039008 & 0.000754 & $0.144603^{*}$ & $-0.002^{* *}$ & $0.117774^{*}$ \\
\hline & {$[0.02140]$} & [ 5.10244] & {$[0.10896]$} & [ 5.53877] & {$[0.01894]$} & {$[0.24473]$} & [ 1.10536$]$ & [ 8.37869] & {$[-2.03343]$} & [ 5.06389] \\
\hline \multirow[t]{2}{*}{ VOLUME(-3) } & 0.00202 & $0.087432 *$ & -0.0 & $0.090703^{*}$ & $-0.0134^{* *}$ & 767 & -0.000763 & 0.090683* & 0.000563 & $0.079863^{*}$ \\
\hline & [ 1.64180] & {$[4.88846]$} & {$[-0.41428]$} & [ 5.23463] & {$[-2.35174]$} & {$[-1.47918]$} & {$[-1.11263]$} & [ 5.22667] & {$[0.56904]$} & [ 3.41611] \\
\hline \multirow[t]{2}{*}{ VOLUME(-4) } & $-0.0040^{*}$ & $0.101323^{*}$ & $-0.00128^{* * *}$ & $0.111336^{*}$ & 0.000844 & 0.230745 & -0.000282 & 0.09149* & 0.000572 & $0.067888^{*}$ \\
\hline & {$[-3.26545]$} & [ 5.66503] & [-1.74149] & [6.42896] & [0.13831] & [ 1.44419] & {$[-0.41133]$} & [ 5.26899] & {$[0.57801]$} & [ 2.90302] \\
\hline \multirow[t]{2}{*}{ VOLUME(-5) } & -0.00050 & $0.086676^{*}$ & $0.001446 * * *$ & $0.123284 *$ & 0.00663 & 0.075975 & $0.002007^{*}$ & $0.12558^{*}$ & -0.00096 & $0.081257^{*}$ \\
\hline & {$[-0.41186]$} & [ 4.83330] & [ 1.95626$]$ & [ 7.11700$]$ & [ 1.12410$]$ & [ 0.49178] & [ 2.94252] & [ 7.27126] & {$[-0.97170]$} & [ 3.49139] \\
\hline \multirow[t]{2}{*}{ VOLUME(-6) } & 0.00132 & $0.087377^{*}$ & 0.000537 & $0.064661 *$ & -0.001741 & 0.148814 & $-0.00118^{* * *}$ & $0.066357^{*}$ & 0.000631 & $0.044425^{* *}$ \\
\hline & [ 1.18037] & [ 5.38769] & {$[0.78645]$} & [ 4.04245] & {$[-0.30827]$} & [ 1.00595$]$ & {$[-1.86938]$} & [ 4.12803] & [ 0.67989] & [ 2.02541] \\
\hline F-Values & 260.956 & 8.273 .011 & 6.386 .239 & 625.693 & 1.467 .092 & 2.778 .519 & 1.691 .059 & 7.183 .415 & 2.932 .152 & 9.008 .137 \\
\hline
\end{tabular}




\begin{tabular}{|c|c|c|c|c|c|c|c|c|c|c|}
\hline & \multicolumn{2}{|c|}{ Ultra tech } & \multicolumn{2}{|c|}{ Infosys tech } & \multicolumn{2}{|c|}{ Maruti Suzuki } & \multicolumn{2}{|c|}{ S\&P CNX Nifty } & \multicolumn{2}{|c|}{ Reliance Infra } \\
\hline & RETURN & VOLUME & RETURN & VOLUME & RETURN & VOLUME & RETURN & VOLUME & RETURN & VOLUME \\
\hline \multirow[t]{2}{*}{ C } & -0.00732 & $3.318444^{*}$ & $0.028221^{*}$ & $1.011097^{*}$ & -0.01211 & $0.924864^{*}$ & -0.002931 & $0.446089 *$ & -0.000574 & $0.459367^{*}$ \\
\hline & {$[-0.85670]$} & [ 10.7729] & [ 3.63634] & [ 7.17971] & {$[-1.53814]$} & [5.51639] & {$[-0.48707]$} & [4.14955] & {$[-0.12394]$} & 5.09969] \\
\hline \multirow[t]{2}{*}{ RETURN(-1) } & 0.02069 & 1.092 .942 & $0.085857^{*}$ & 0.237806 & $0.033771 * * *$ & -0.684521 & $0.063829 *$ & $0.782118^{*}$ & $0.037235^{* *}$ & $0.65331^{* *}$ \\
\hline & [ 0.93697$]$ & [ 1.37297] & [ 5.31344] & [ 0.81103$]$ & [1.65920] & {$[-1.57933]$} & [ 3.95161] & [ 2.71075] & [ 2.29329] & [ 2.07018] \\
\hline \multirow[t]{2}{*}{ RETURN(-2) } & -0.029112 & $3.153472^{*}$ & $-0.055051^{*}$ & $-1.031703^{*}$ & -0.016098 & -0.056407 & $-0.046584^{*}$ & 0.285139 & $-0.048184 *$ & -0.072476 \\
\hline & {$[-1.32138]$} & [ 3.97042] & {$[-3.39787]$} & {$[-3.50922]$} & {$[-0.7$} & {$[-0.13007]$} & {$[-2.87866]$} & [ 0.98644] & {$[-2.96$} & 43] \\
\hline \multirow[t]{2}{*}{ RETURN(-3) } & 0.026963 & $2.513122^{*}$ & -0.01 & & -0.0 & -0.0 & 0.0 & 367 & & \\
\hline & [ 1.22 & {$[3$.} & {$[-0.6$} & & & {$[-0$.} & & & & \\
\hline \multirow[t]{2}{*}{ RETURN(-4) } & -0.025108 & -0.0 & -0.026228 & $-0.49634 * * *$ & & 0.2 & & & & \\
\hline & {$[-1.13284]$} & {$[-0.06212]$} & {$[-1.61431]$} & {$[-1.68350]$} & {$[0.53798]$} & {$[0.53452]$} & {$[0.46800]$} & {$[0.01406]$} & {$[-1.42396]$} & {$[-0.46316]$} \\
\hline \multirow[t]{2}{*}{ RETURN(-5) } & $0.060474^{*}$ & & -0.0 & & & & & $9^{* *}$ & & \\
\hline & {$[2.72$} & {$[-1.6$} & {$[-0.5$} & {$[-0$.} & {$[-1$.} & {$[-1$.} & {$[-0]$.} & 43] & {$[-0$.} & \\
\hline \multirow[t]{2}{*}{ RETURN(-6) } & -0.01 & 91 & -0.03 & & & 72 & -0.04 & 556 & -0.0 & 27 \\
\hline & {$[-0.46325]$} & [0.54899] & {$[-2.40113]$} & {$[0.56557]$} & {$[-0.93888]$} & {$[0.82928]$} & {$[-2.70243]$} & {$[-0.59744]$} & {$[-0.41030]$} & {$[0.7$} \\
\hline \multirow[t]{2}{*}{ RETURN(-7) } & - & 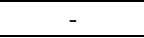 & $-0.02933^{* * *}$ & -0.055741 & 0.005741 & -0.115947 & 0.012508 & -0.085032 & 97 & -0.331074 \\
\hline & - & - & {$[-1.8$} & & & & & & & \\
\hline \multirow[t]{2}{*}{ VOLUME(-1) } & -0.000153 & $0.276347^{*}$ & -0.0 & 0.42 & & $0.453863^{*}$ & $0.002403^{*}$ & $0.389475^{*}$ & 0.0017 & 0.46 \\
\hline & {$[-0.24$} & {$[12$.} & {$[-0.5$} & {$[26$} & {$[0 . \varepsilon$} & [22.3556] & {$[2.6$} & [ 24.1257] & [ 2.12066$]$ & {$[2$} \\
\hline \multirow[t]{2}{*}{ VOLUME(-2) } & -0.000174 & $0.175432^{*}$ & $-0.002107^{* *}$ & $0.093634^{*}$ & -0.0 & $0.095114^{*}$ & -0.001268 & $0.154505^{*}$ & -0.00 & $0.132276^{*}$ \\
\hline & {$[-0.27613]$} & [ 7.72245] & {$[-2.18128]$} & [ 5.34091] & {$[-0.29709]$} & [4.26665] & {$[-1.30677]$} & [ 8.91329] & {$[-1.07545]$} & [ 7.40493] \\
\hline \multirow[t]{2}{*}{ VOLUME(-3) } & -0.000557 & $0.086578^{*}$ & $0.002459 * *$ & 0.099263* & 0.001454 & $0.097802^{*}$ & 0.001572 & $0.098896 *$ & -0.000272 & $0.101724^{*}$ \\
\hline & {$[-0.87$} & [ 3.75836$]$ & {$[2.53$} & & & & & & {$[-0$.} & \\
\hline \multirow[t]{2}{*}{ VOLUME(-4) } & -0.00002 & & -0.00 & $0.165359 *$ & -0.0 & $0.095276^{*}$ & -0.0 & 0.116 & 0.000 & 0.07 \\
\hline & {$[-0.02685]$} & {$[0.90258]$} & {$[-1.08$} & [9.47203] & {$[-0.9$} & [4.27839] & {$[-0.43458]$} & [6.71263] & {$[0.40122]$} & {$[4.26$} \\
\hline \multirow[t]{2}{*}{ VOLUME(-5) } & $0.001252^{* *}$ & $0.121742^{*}$ & -0.000375 & $0.061913^{*}$ & -0.000494 & $0.097126^{*}$ & -0.001238 & $0.127037^{*}$ & -0.000757 & $0.101914^{*}$ \\
\hline & {$[1.99470]$} & {$[5.37870]$} & {$[-0.38690]$} & [ 3.51835] & {$[-0.47208]$} & [ 4.35991] & {$[-1.27133]$} & [ 7.30208] & {$[-0.82114]$} & [ 5.68847] \\
\hline \multirow[t]{2}{*}{ VOLUME(-6) } & 0.000366 & 0.026681 & -0.000522 & 0.026697 & $0.001921 * * *$ & 0.012204 & 0.000756 & $0.035092^{* *}$ & 0.000548 & $0.042862^{* *}$ \\
\hline & [0.60149] & [ 1.21513] & {$[-0.54008]$} & [1.52349] & [1.83656] & {$[0.54779]$} & {$[0.77876]$} & [ 2.02307] & {$[0.59668]$} & [ 2.39928] \\
\hline \multirow[t]{2}{*}{ VOLUME(-7) } & - & - & 0.000202 & 0.05 & & $0.081086^{*}$ & $-0.00161^{* * *}$ & $0.053699 *$ & -0.000611 & 0.0439 \\
\hline & - & & {$[0.2$} & 33] & {$[-1$.} & 50] & {$[-1.78436]$} & 2405] & {$[-0.73226]$} & [2.70992] \\
\hline r-values & 1.471 .046 & 6.777 .792 & 5.070 .115 & 680.543 & 1.175 .827 & 4.395 .776 & 3.172 .081 & 1.943 .485 & 1.642 .663 & 1460.77 \\
\hline
\end{tabular}


Table 4g: Vector Autoregression Model Results

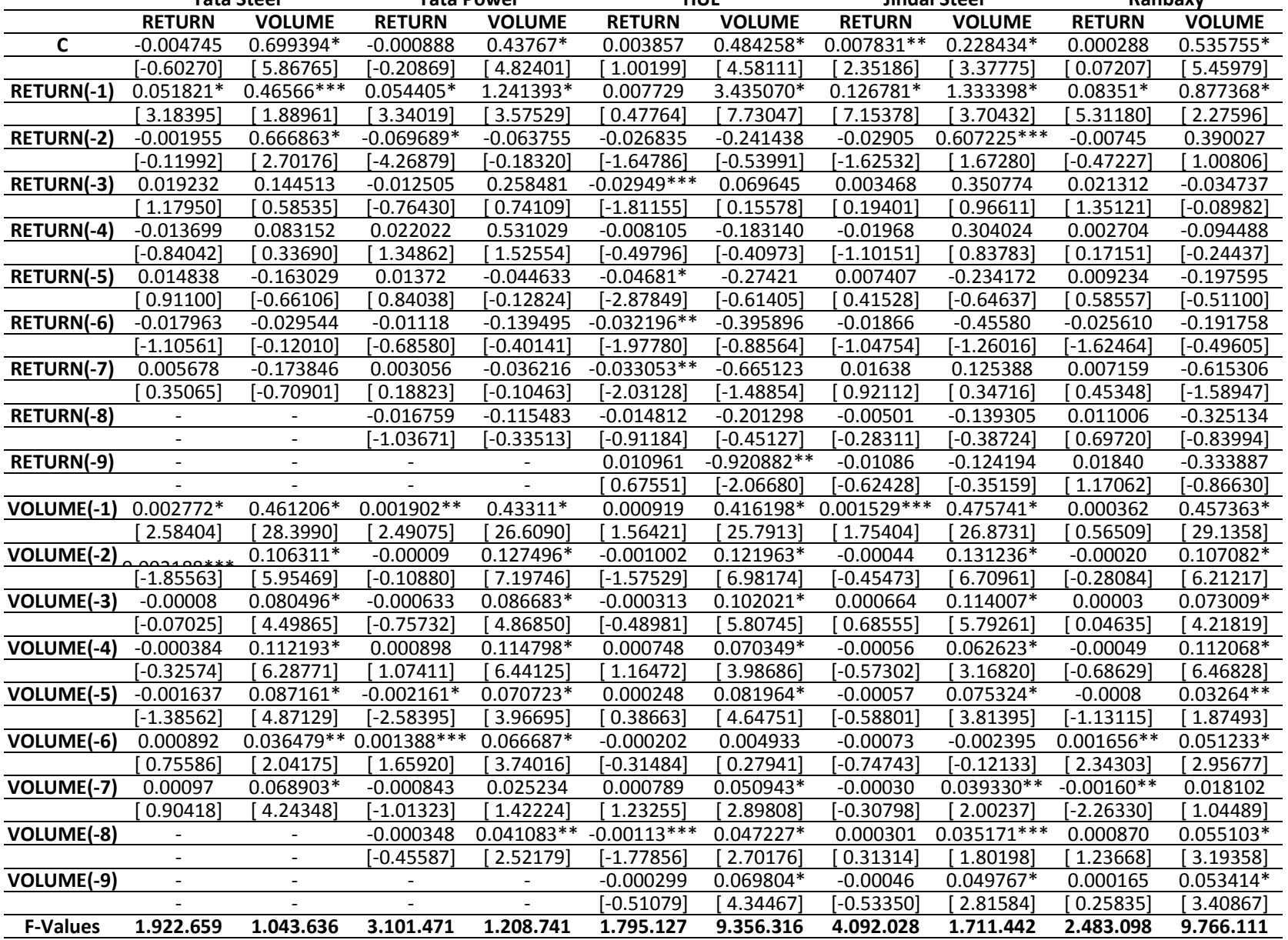




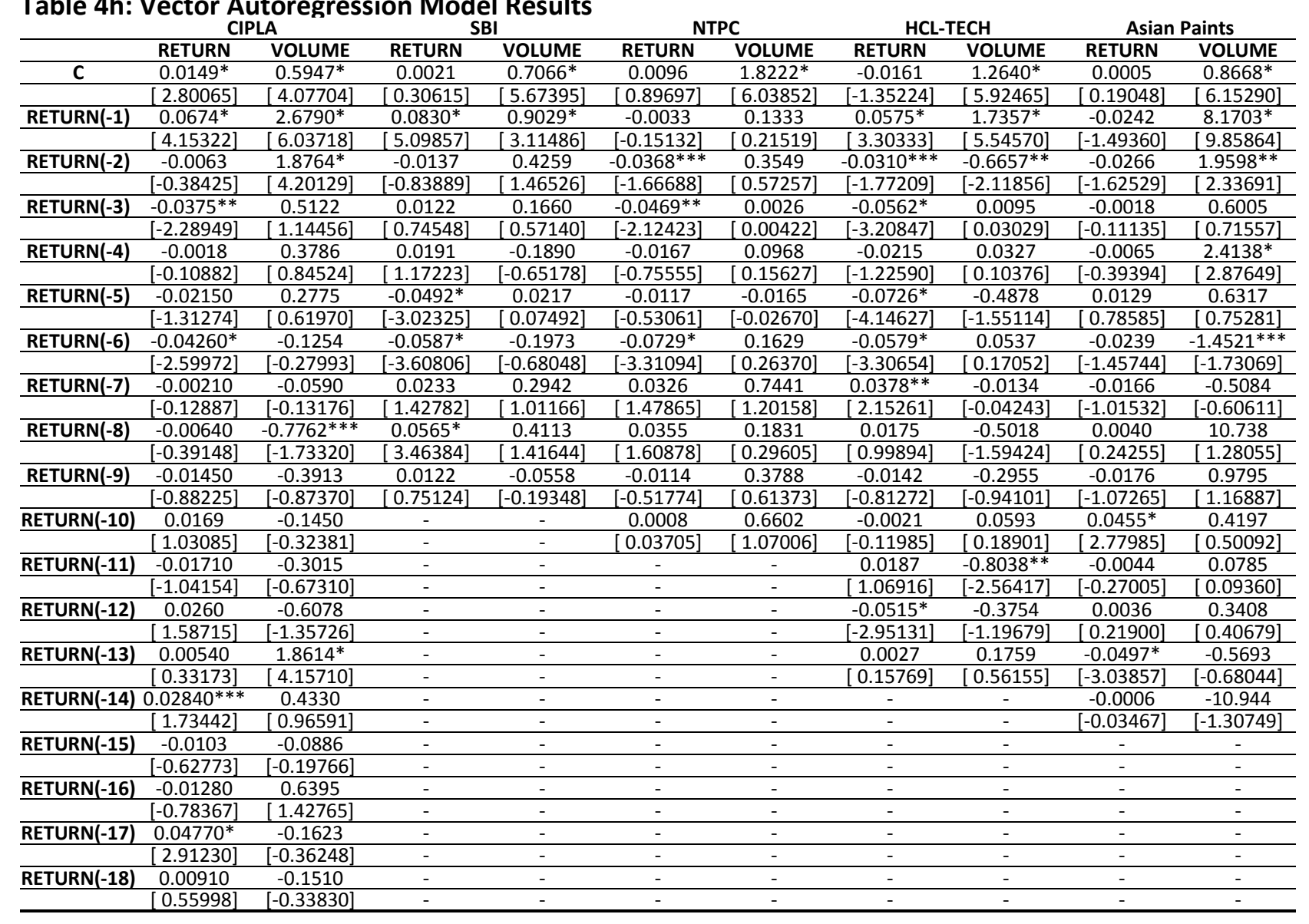




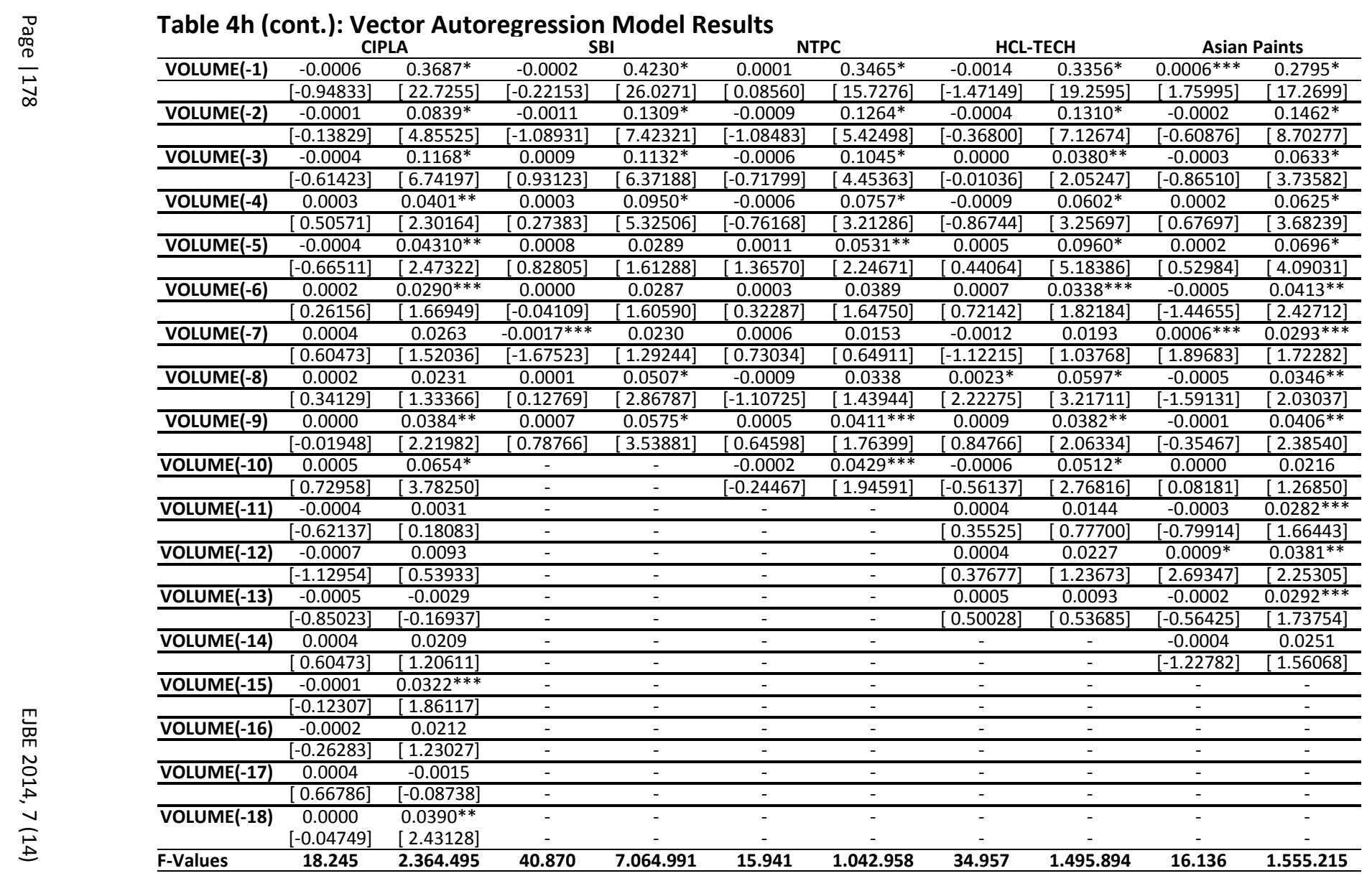


Investigation of Causal Relationship between Stock Prices and Trading Volume using...

It is relevant to mention here that results shown by the VAR modeling are marginally different from the results shown by Toda and Yamamoto Granger Causality tests. The possible reason behind the dissimilar results could be the different theoretical background of the two methodologies. Although results are little different, nevertheless presence of interaction between return and trading volume and significant role of stock returns cannot be denied.

\section{Concluding Remarks}

Monitoring stock prices and trading volume simultaneously can be very prolific for the dynamics of price discovery process. Previous studies (irrespective of their sample size or economy i.e. developing/developed) have already shown how indispensable is the study of above mentioned variables to know the future movements of financial markets. Taking clue from this, present study investigates the empirical relationship between stock returns and trading volume by using daily data of 39 individual securities and S\&P CNX Nifty from January 1, 1998 to May 31, 2013. All possible precautions have been considered during the analysis of data, such as (i) instead of applying ordinary Granger causality test; Toda and Yamamoto (1995) test has been used (ii) lag length chosen by AIC and FPE criterion has further been insured by running Lagrange Multiplier (LM) test (iii) Causality determined by Toda and Yamamoto test has also been confirmed by using the VAR model.

Though results shown by Toda and Yamamoto and VAR test were little dissimilar, the empirical analysis provides sufficient grounds to declare the presence of interaction (either unilateral/bilateral) between stock returns and trading volume. However, some securities have shown independent movement in the stock returns and trading volume without communicating each other. As far as lead/lag relationship is concerned, around 80 per cent (i.e. 31 securities) of the individual securities have declared stock return 'a motivating force' behind investments. However, 13 per cent of the individual securities (i.e. 5 securities) and an index have shown the deterministic role of trading volume over stock returns. The policy implications of our findings are straightforward i.e. steps must be taken to stabilize the stock prices so that investors' decisions can be made more rationalized.

\section{References}

Akaike, H. (1974) "A New Look at the Statistical Model Identification." IEEE Trans. Autom. Control 19:716-723.

http://dx.doi.org/10.1109/TAC.1974.1100705

Bhagat, S. \& Bhatia, S. (1996) "Trading Volume and Price Variability: Evidence on Lead-lag Relations from Granger-Causality Tests." Working Paper, University of Colorado at Boulder.

Brooks, C. (2002), "Multivariate Models." Introductory Econometrics for Finance (3rd Ed.) pp 302-315 Cambridge: Cambridge University Press.

Blume, L., D. Easley \& Hara, M.O. (1994) "Market Statistics and Technical Analysis: The Role of Volume." Journal of Finance 49 (1):153-181.

http://dx.doi.org/10.1111/j.1540-6261.1994.tb04424.x

EJBE 2014, 7 (14)

Page | 179 
Campbell. Y. J., Grossman, S. J. \& Wang, J. (1993) "Trading Volume and Serial Correlation in Stock Returns." Quarterly Journal of Economics 108 (4): 905- 939. http://dx.doi.org/10.2307/2118454

Chen, G. M. \& Rui, M.O. (2001) "The Dynamic Relation between Stock Return, Trading Volume and Volatility." The Financial Review 38:153-174. http://dx.doi.org/10.1111/j.1540-6288.2001.tb00024.x

Chordia, T. \& Swaminathan, B. (2000) "Trading Volume and Cross-Autocorrelations in Stock Returns." Biography Center SSRN: http://ssrn.com/abstract=157835 (accessed September 28, 2013).

Crouch, R. L. (1970) "The Volume of Transactions and Price Changes of the New York Stock Exchange." Financial Analysts Journal $26 \quad$ (4): 104-109. http://dx.doi.org/10.2469/faj.v26.n4.104

Darwish, M. (2012) "Testing the Contemporaneous and Causal Relationship between Trading Volume and Return in the Palestine Exchange." International Journal of Economics and Finance 4 (4):182-192.

http://dx.doi.org/10.5539/ijef.v4n4p182

De Medeiros, Otavio R., Doornik, V. \& Bernardus F. N. (2006) "The Empirical Relationship between Stock Returns, Volatility and Trading Volume in the Brazilian Stock Market." Biography Center SSRN: http://ssrn.com/abstract=897340 (accessed on October 12, 2013).

Dickey, D.A. \& Fuller, W.A (1979) "Distribution of the Estimators for Autoregressive Time Series with a Unit Root." Journal of American Statistical Association 74: 427-431. http://dx.doi.org/10.2307/2286348

Godfrey, M. D., Granger, J.W.C. \& Morgenstern, O. (1964) "The Random Walk Hypothesis of Stock Market Behavior." Kyklos 17: 1-30.

http://dx.doi.org/10.1111/j.1467-6435.1964.tb02458.x

Granger, C. W. J. \& Morgenstern, O. (1963) "Spectral Analysis of New York Stock Market Prices." Kyklos 16 (1): 1-27.

http://dx.doi.org/10.1111/j.1467-6435.1963.tb00270.x

Granger, C W J. (1969) "The Combination of Forecasts." Operational Research Quarterly 20: 451-468.

http://dx.doi.org/10.1057/jors.1969.103

Gujarati, D. N. \& Sangeetha (2007) "Dynamics Econometrics Models: Autoregressive and Distributed - Lag Models. Basic Econometrics." Basic Econometrics (4 ${ }^{\text {th }}$ Ed.) pp $714 \mathrm{New}$ Delhi: Tata McGraw Hill.

Gujarati, D. N. and Sangeetha (2007) "Time Series Econometrics: Some Basic Concepts." Basic Econometrics (4 ${ }^{\text {th }}$ Ed.) pp. 811-839 New Delhi: Tata McGraw Hill.

Hiemstra, G. \& Jones, D.J. (1994) "Testing for Linear and Nonlinear Granger Causality in the Stock Price-Volume Relation." The Journal of Finance XLIX (5): 1639-1663. http://dx.doi.org/10.2307/2329266

Karpoff, J. (1987) "The Relation between Price Changes and Trading Volume: A survey." Journal of Financial and Quantitative Analysis 22: 109-126. http://dx.doi.org/10.2307/2330874

Llorente, G., R. Michaely, G. S. \& Wang, J. (2002) "Dynamic Volume-Return Relation of Individual Stocks." The Review of Financial Studies 15 (4): 1005-1047. http://dx.doi.org/10.1093/rfs/15.4.1005 
Mahajan, Sarika \& Singh, Balwinder (2009) "The Empirical Investigation of Relationship between Return, Volume and Volatility Dynamics in Indian Stock Market." Eurasian Journal of Business and Economics 2(4): 113-137.

Martikainen, T., V. Puttonen, M. L. \& Rothovius, T. (1994) "The Linear and Non-Linear Dependence of Stock Returns and Trading Volume in the Finnish Stock Market." Applied Financial Economics 4: 159-169.

http://dx.doi.org/10.1080/758523960

Mehrabanpoor, M., Bahador, B. V. \& Jandaghi, G. (2011) "Stock Exchange Indices and Turnover Value-Evidence from Tehran Stock Exchange." African Journal of Business Management 5 (3): 783-791.

Phillips, P.C.B. \& Perron, P. (1988) "Testing for a Unit Root in Time Series Regression." Biometrika 75:335-346.

http://dx.doi.org/10.1093/biomet/75.2.335

Ratner. M. \& Leal, R.P.C. (2001) "Stock Returns and Trading Volume: Evidence from the Emerging Markets of Latin America and Asia." Journal of Emerging Markets. 6 (1): 5-22.

Sabri, N. R. (2008) "The Impact of Trading Volume on Stock Price Volatility in the Arab Economy." Biography Center SSRN: http://ssrn.com/abstract=1097624 (accessed on November 20, 2013).

Saatcioglu, K. \& L. Starks (1998) "The Stock Price-Volume Relationship in Emerging Stock Markets: The Case of Latin America." International Journal of Forecasting 14: 215-225. http://dx.doi.org/10.1016/S0169-2070(98)00028-4

Schwartz, G. (1978) "Estimating the Dimension of a Model." The Annals of Statistics 5: 461464.

Smirlock, M. \& Starks, L. (1988) “An Empirical Analysis of Stock Price-Volume Relationship." Journal of Banking and Finance 12: 31-41. http://dx.doi.org/10.1016/0378-4266(88)90048-9

Tripathy, N. (2011) "The Relation between Price Changes and Trading Volume: A Study in Indian Stock Market." Interdisciplinary Journal of Research in Business 1 (7): 81-95.

Zolotoy L. \& Melenberg B. (2007) "Trading Volume, Volatility and Return Dynamics: Individual and Cross-Market Analysis." Biography Center SSRN: http://ssrn.com/abstract=1032193 (accessed on October 10, 2013). 